\title{
Der Informationsgehalt des Betreibungsregisters: Ein Reformvorschlag zu seiner Verbesserung
}

Urs Boller / Mark Schweizer *

Ungerechtfertigte Betreibungen können für den Betroffenen nachteilige Folgen haben, weil sie auch dann im Betreibungsregisterauszug erscheinen, wenn sie vom Gläubiger nicht weiterverfolgt werden. Ein Gesetzesvorschlag zur Revision des SchKG will Abhilfe schaffen. Aufgrund schematischer Kriterien sollen bestimmte Betreibungen vom Einsichtsrecht ausgeschlossen werden. Die Autoren bezweifeln, dass dieser Vorschlag den Informationsgehalt des Betreibungsregisters verbessert. Sie schlagen stattdessen vor, den Anwendungsbereich der Klage nach Art. 85 SchKG so zu erweitern, dass ungerechtfertigte Betreibungen aufgehoben werden können, ohne dass der Betriebene ein hohes Kostenrisiko trägt.

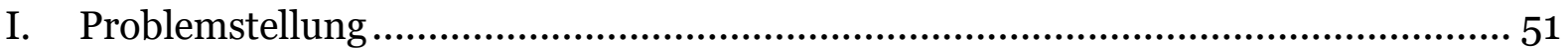

II. Einleitung des Betreibungsverfahrens und Einsicht in das Betreibungsregister ......52

1. Einleitung des Betreibungsverfahrens ohne Prüfung der Forderung ....................52

2. Betreibungsregister und Betreibungsauskunft .............................................. 53

III. Informationswert des Betreibungsregisters......................................................... 54

IV. Möglichkeiten zur Aufhebung ungerechtfertigter Betreibungen de lege lata ...........59

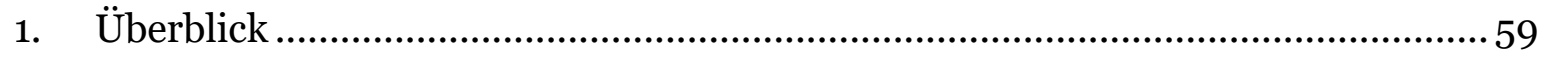

2. Klage auf Aufhebung oder Einstellung der Betreibung im ordentlichen oder vereinfachten Verfahren nach Art. 85a SchKG ......................59

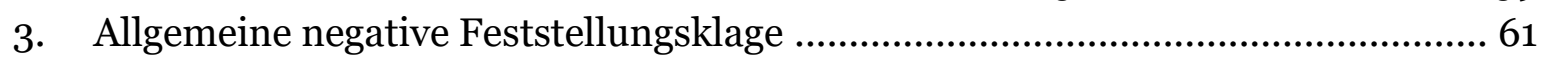

4. Klage auf Aufhebung oder Einstellung der Betreibung im summarischen Verfahren nach Art. 85 SchKG

5. Geltendmachung der Nichtigkeit der Betreibung mittels betreibungsrechtlicher

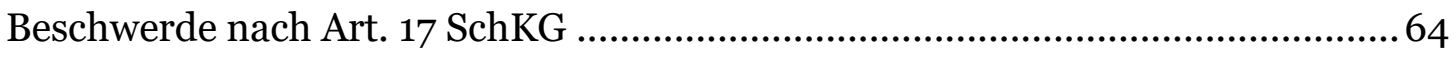

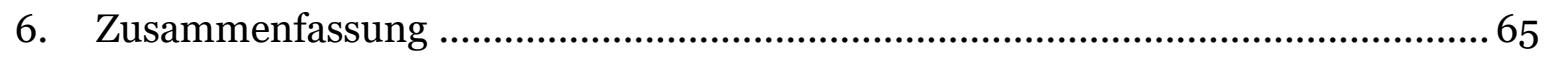

V. Der Gesetzesvorschlag der Kommission für Rechtsfragen des Nationalrats ...........66

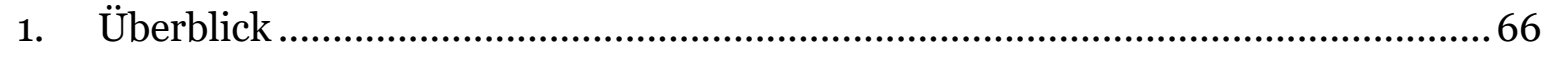

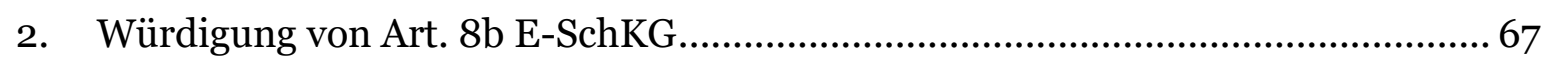

VI. Vorschlag der Autoren: Ausbau der Klage nach Art. 85 SchKG ............................ 71

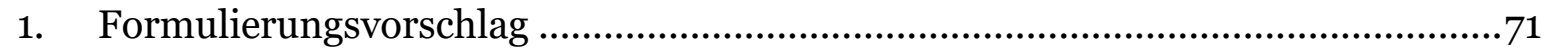

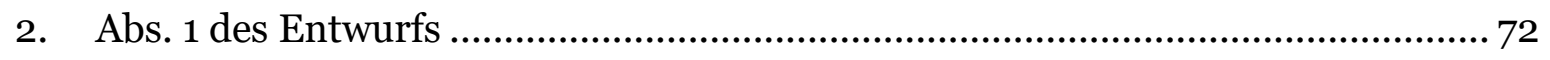

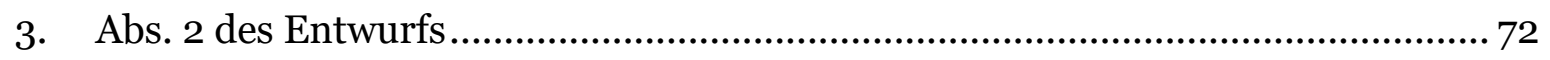

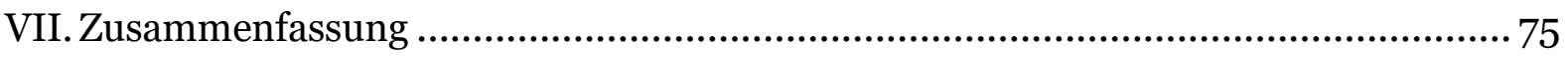

Zitiervorschlag: Urs Boller / Mark Schweizer, Der Informationsgehalt des Betreibungsregisters: Ein Reformvorschlag zu seiner Verbesserung, in: sui-generis 2015, S. 50

URL: $\quad$ sui-generis.ch/13

DOI: $\quad$ https://doi.org/10.21257/sg.13 


\section{Problemstellung}

1 Nach schweizerischem Recht kann grundsätzlich jede Person ein Zwangsvollstreckungsverfahren auf Bezahlung einer Geldschuld einleiten, ohne dass geprüft würde, ob die Forderung tatsächlich besteht. Im Gegenzug kann der angebliche Schuldner die Zwangsvollstreckung durch einfache Erklärung («Rechtsvorschlag») zum Stillstand bringen. Problematisch ist dabei, dass das von der Zwangsvollstreckungsbehörde (Betreibungsamt) geführte Register auch Auskunft über eingeleitete, aber nicht fortgesetzte, Betreibungen gibt. Da der Auszug aus dem Betreibungsregister in der Schweiz die wichtigste Urkunde zum Nachweis der Zahlungsfähigkeit ist, können ungerechtfertigte Einträge das wirtschaftliche Fortkommen der betroffenen Person erheblich beeinträchtigen.

Es ist umstritten, wie oft ungerechtfertigte Betreibungen in der Praxis tatsächlich vorkommen. Der Initiant einer parlamentarischen Initiative vom 11. Dezember 2009 zur raschen Löschung ungerechtfertigter Zahlungsbefehle, Fabio Abate, geht davon aus, dass solche missbräuchlichen Betreibungen («Schikanebetreibungen») häufig vorkommen und fordert Abhilfe. ${ }^{1}$ Der auf die Initiative Abate hin ausgearbeitete Vorschlag für eine Gesetzesänderung der Kommission

* Urs Boller, LL.M. (Queen Mary, London), MClArb, Rechtsanwalt in Zürich.

Mark Schweizer, PD Dr. iur., LL.M. (Ann Arbor), Rechtsanwalt in Zürich.

Dieses Werk ist lizenziert unter einer Creative Commons Namensnennung - Weitergabe unter gleichen Bedingungen 4.o International Lizenz.

09.530 - Parlamentarische Initiative - Abate Fabio - Löschung ungerechtfertigter Zahlungsbefehle. für Rechtsfragen des Nationalrats vom 19. Februar $2015^{2}$ sieht eine Beschränkung des Einsichtsrechts Dritter in das Betreibungsregister betreffend hängiger Betreibungen vor, in denen der Rechtsvorschlag nicht beseitigt ist. Auf Antrag des Betriebenen gibt das Betreibungsamt Dritten vorläufig keine Kenntnis von solchen Betreibungen (Art. 8b Abs. 1 ESchKG). Solche hängigen Betreibungen sollen Dritten nur noch mitgeteilt werden, wenn seit der Einleitung der Betreibung und in den sechs Monaten davor vor dem gleichen Amt Betreibungen von mindestens zwei weiteren Gläubigern eingeleitet worden sind (Art. 8b Abs. 2 lit. a E-SchKG), oder wenn in den letzten zwölf Monaten gegen den Schuldner eine Betreibung fortgesetzt wurde (Art. 8b Abs. 2 lit. b E-SchKG), oder wenn in den letzten zwölf Monaten eine betriebene Forderung durch Zahlung an das Betreibungsamt beglichen wurde und der Gläubiger die Betreibung nicht zurückgezogen hat (Art. 8b Abs. 2 lit. c E-SchKG).

3 Der neue Art. 8b E-SchKG ist innerhalb der nationalrätlichen Kommission umstritten. Eine Minderheit will auf den neuen Artikel verzichten. 3 Bereits in der Vernehmlassung wurde kritisiert, dass durch die vorgeschlagene Regelung der Informationswert des Betreibungsregisterauszugs beeinträchtigt würde, da auch gerechtfertigte Betreibungen nicht mehr erschienen.4 An ähnlichen Bedenken war

2 Gesetzesvorschlag vom 19. Februar zu einer Änderung des Bundesgesetzes über Schuldbetreibung und Konkurs (nachfolgend «E-SchKG»), BBl 20153223.

3 Bericht der Kommission für Rechtsfragen des Nationalrates vom 19. Februar 2015, BBl 2015 3209 (im Folgenden: «Bericht der Kommission»), 3216.

4 Bericht des Bundesamts für Justiz über das Ergebnis des Vernehmlassungsverfahrens zu 
bereits die parlamentarische Initiative Jean Studer 2007 gescheitert, die verlangte, eingestellte Betreibungen Dritten nicht bekannt zu geben. 5

In diesem Aufsatz versuchen wir uns dem Thema Informationswert des Betreibungsregisters grundsätzlich zu nähern und untersuchen, ob der auf der parlamentarischen Initiative Abate basierende Gesetzesvorschlag geeignet ist, den Informationswert des Registers zu erhöhen. Nach der einleitenden Problemstellung (I.) werden das Verfahren zur Einleitung der Betreibung und die Einsicht in das Betreibungsregister nach geltendem Recht dargestellt (II.). Anschliessend wird untersucht, unter welchen Umständen dem Betreibungsregister wertvolle Informationen zur Zahlungswilligkeit und Zahlungsfähigkeit des Betriebenen entnommen werden können, wenn der Rechtsvorschlag (noch) nicht beseitigt wurde (III.). Im vierten Teil wird in gebotener Kürze gezeigt, welche Möglichkeiten zur Aufhebung ungerechtfertigter Betreibungen unter geltendem Recht zur Verfügung stehen (IV.). Sodann wird der Gesetzesvorschlag der Rechtskommission des Nationalrats auf seine Auswirkungen auf den Informationswert des Betreibungsregisters überprüft (V.). In einem letzten Teil machen wir einen eigenen Vorschlag, wie das Problem de lege ferenda gelöst werden könnte (VI.).

09.530 - Parlamentarische Initiative Abate (nachfolgend «Vernehmlassungsbericht»), $3 \mathrm{ff}$. 04.467 - Parlamentarische Initiative - Studer Jean - Keine Veröffentlichung eingestellter Betreibungen, Bericht der Kommission für Rechtsfragen vom 6. November 2007; siehe dazu auch den Tätigkeitsbericht des Eidgenössischen Datenschutzbeauftragten 2007/8, Die Aussagekraft von Betreibungsregisterauszügen.

\section{Einleitung des Betreibungsverfah- rens und Einsicht in das Betrei- bungsregister}

\section{Einleitung des Betreibungsverfah- rens ohne Prüfung der Forderung}

5 Das schweizerische Recht zeichnet sich dadurch aus, dass der Gläubiger ein Verfahren zur Vollstreckung einer Geldforderung einleiten kann, ohne dass er den Bestand seiner Forderung belegen muss. ${ }^{6}$ Das Betreibungsamt prüft den vom Gläubiger behaupteten Anspruch nicht auf seine materielle Begründetheit hin.7 Vorbehältlich eines offensichtlich rechtsmissbräuchlichen Betreibungsbegehrens hat das Betreibungsamt den Zahlungsbefehl gemäss den Angaben des Gläubigers auszustellen. ${ }^{8}$ Entsprechend können Betreibungen angehoben werden, ohne dass dem Betreibenden eine Forderung gegen den Betriebenen zusteht. Andererseits kann der Betriebene die Zwangsvollstreckung in sein Vermögen durch eine einfache Erklärung durch Erhebung des Rechtsvorschlags ohne weiteres zum Stillstand bringen.9 Will der Gläubiger die Betreibung fortsetzen, so hat er den Rechtsvorschlag des Schuldners beseitigen zu lassen. Oftmals befasst sich erst in diesem Stadium ein Gericht oder eine Verwaltungsbehörde

6 Vgl. statt vieler BGE 125 III 149 E. 2a.; Botschaft über die Änderung des Bundesgesetzes über Schuldbetreibung und Konkurs (SchKG) vom 8. Mai 1991, BBl 1991 III 1 (nachfolgend «Botschaft SchKG»), 6.

7 Statt aller BGE 110 III 20 E. 2.

8 BGer, Urteil 5A_582/2009 vom 26. November 2009, E. 3.1. Zur Frage des Rechtsmissbrauchs siehe hierzu hinten, Ziff. 5.

9 Art. 74 Abs. 1 und 78 Abs. 1 SchKG. Der Rechtsvorschlag braucht im Normalfall nicht begründet zu werden (Art. 75 Abs. 1 SchKG), ausser etwa beim nachträglichen Rechtsvorschlag (Art. 77 Abs. 2 SchKG) sowie beim Rechtsvorschlag in der Wechselbetreibung (Art. 179 Abs. 1 SchKG). 
mit der Frage, ob die betriebene Forderung wirklich besteht, ${ }^{10}$ oder ob sich der Gläubiger zumindest auf eine Schuldanerkennung oder auf eine vollstreckbare öffentliche Urkunde stützen kann. ${ }^{11}$ Davon zu unterscheiden sind die Fälle, in denen der Gläubiger mit der Betreibung die Vollstreckung eines Entscheids bezweckt: Hier hat bereits vor Einleitung der Betreibung eine materielle Anspruchsprüfung stattgefunden, der Gläubiger wird hier also in aller Regel den Schuldner zu Recht betreiben. ${ }^{12}$

\section{Betreibungsregister und Betrei- bungsauskunft}

6 Die Betreibungsämter führen über ihre Amtstätigkeit Protokoll (sog. «Betreibungsregister»). ${ }^{13} \mathrm{Zu}$ den vom Betreibungsamt geführten Registern gehört insbesondere das «Betreibungsbuch», in das sämtliche Betreibungen eingetragen werden. Primäre Funktion des Betreibungsregisters ist, die Amtstätigkeit des Betreibungsamtes $\mathrm{zu}$ dokumentieren, damit das Amt Rechenschaft über seine Tätigkeit ablegen kann. ${ }^{14}$

7 In der Praxis von kaum zu überschätzender Bedeutung ist die sekundäre Funktion des Betreibungsregisters. Diese besteht darin, Dritten Informationen zur Zahlungsfähigkeit und Zahlungswilligkeit

10 Im Anerkennungsprozess nach Art. 79 SchKG.

11 Im Rechtsöffnungsverfahren nach Art. 80 ff. SchKG.

12 Dementsprechend sind denn auch die Verteidigungsmöglichkeiten des Schuldners im Rechtsöffnungsverfahren stark eingeschränkt (vgl. Art. 81 Abs. 1 SchKG).

13 Art. 8 Abs. 1 SchKG sowie im Einzelnen Art. 8-15 der Verordnung über die im Betreibungs- und Konkursverfahren zu verwendenden Formulare und Register sowie die Rechnungsführung (VFRR, SR 281.31).

14 Dominik Gasser, Revidiertes SchKG - Hinweise auf kritische Punkte, ZBJV 1996, 627, 630. des Betriebenen zu liefern.15 Anspruch auf Einsicht und auf den Erhalt von Auszügen hat jede Person, die ein Interesse daran glaubhaft machen kann (Art. 8a SchKG). Art. 8a Abs. 2 SchKG erwähnt den Fall, dass das Auskunftsgesuch in unmittelbarem Zusammenhang mit dem Abschluss oder der Abwicklung eines Vertrages gestellt wird. In der Praxis wird häufig der potentielle Vertragspartner aufgefordert, selbst einen Betreibungsregisterauszug zu seiner Person vorzulegen. Obwohl keine Rechtspflicht besteht, einem solchen Ansinnen Folge $\mathrm{zu}$ leisten, wird eine Weigerung in der Regel dahingehend verstanden, dass die betroffene Person «etwas zu verbergen» hat, also Einträge im Betreibungsregister vorhanden sind.

8 Die mutmassliche Zahlungsfähigkeit und Zahlungswilligkeit und damit Kreditwürdigkeit einer Person kann bedeutsam sein für die Entscheidung, ob mit der fraglichen Person ein Geschäft abgeschlossen werden soll, bei dem der eine Vertragspartner in Vorleistung treten muss oder mit dem ein Dauerschuldverhältnis (wie Miete, Arbeitsvertrag) begründet wird. Ein Betreibungsregister, das nicht «sauber» ist, kann das wirtschaftliche Fortkommen des Betroffenen daher empfindlich einschränken; ${ }^{16}$ gerade in Märkten, in denen Anbieter die Marktmacht haben, wie auf den Wohnungsmärkten in schweizerischen Ballungszentren.

9 Darüber hinaus kann eine Überprüfung der Zahlungsfähigkeit auch nach erfolg-

15 Henry Peter, in: Hunkeler (Hrsg.), Kurzkommentar SchKG, 2. Aufl., Zürich 2014 (nachfolgend «KuKo-SchKG-Bearbeiter»), Art. 8a N 1.

16 Bericht der Kommission (Fn. 3), 3214. 
tem Vertragsabschluss angezeigt sein: Dann nämlich, wenn der Gläubiger entscheiden muss, ob er eine fällige Forderung gegen den zahlungsunwilligen Schuldner durchsetzen will. Dafür fallen meist erhebliche Gerichts- und Anwaltskosten an. Kosten, die sich der Gläubiger sparen kann, wenn er damit rechnen muss, dass die Forderung mangels Zahlungsfähigkeit des Schuldners uneinbringlich sein wird. Die Möglichkeit, die Kreditwürdigkeit des Geschäftspartners anhand des Betreibungsregisters $\mathrm{zu}$ überprüfen, dient der wirtschaftlichen Effizienz und liegt im öffentlichen Interesse. ${ }^{17}$

\section{Informationswert des Betreibungs- registers}

Aufgrund der Möglichkeit der Anhebung einer Betreibung ohne weitergehenden Nachweis der (Gläubiger-)Stellung sowie der Ausgestaltung der Führung des Betreibungsregisters und der Betreibungsauskunft können auch ungerechtfertigte Betreibungen ihren Eingang in das Betreibungsregister finden und der Betreibungsauskunft unterliegen. ${ }^{18}$ Es ist daher näher abzuklären, welchen Wert die im Betreibungsregister zu einer Person verzeichneten Informationen haben. Dies wiederum ist, so die hier vertretene These, entscheidend für das Einsichtsrecht Dritter: Dritte haben nur ein begründetes Interesse daran, Einträge im Betreibungsregister zu kennen, die einen Rückschluss auf die Zahlungsfähigkeit und Zahlungswilligkeit der betroffenen Person zulassen. ${ }^{19}$ Jede vorgeschlagene Re-

17 BGE 115 III 81 E. 2.

18 Bericht der Kommission (Fn. 3), 3212; vgl. ferner statt vieler BGE 128 III 334.

19 Yasmin Iqbal, SchKG und Verfassung - untersteht auch die Zwangsvollstreckung dem Grundrechts- vision des Einsichtsrechts muss daher daraufhin geprüft werden, ob sie den Informationswert des Betreibungsregisters verbessert.

11 Relativ einfach sind die Fälle, in denen ein Betreibungsverfahren formell zu Ende geführt wurde:

1. Kam ein Gläubiger $\mathrm{zu}$ Verlust, ${ }^{20}$ so steht fest, dass der Schuldner zahlungsunfähig ist.

2. Kam zwar niemand zu Verlust, musste aber gleichwohl zur Pfändung und Verwertung geschritten werden, ${ }^{21}$ so ist der Schuldner zumindest nicht zahlungswillig. Auch die Zahlungsfähigkeit dürfte in Frage stehen, wird doch ein solventer Schuldner nicht ohne weiteres eine Pfändung über sich ergehen lassen.

3. Erlosch die Betreibung durch Zahlung des Schuldners an das Betreibungsamt, ${ }^{22}$ so bestehen zumindest Zweifel an der Zahlungswilligkeit des Schuldners, da dieser eine offensichtlich bestehende Forderung erst unter Betreibungsdruck bezahlt hat. 23

12 Dritte haben zweifellos ein berechtigtes Interesse, diese Vorgänge $\mathrm{zu}$ erfahren. Immer noch aussagekräftig erscheint, wenn ein Betreibungsverfahren zwar noch nicht formell abgeschlossen ist, der Gläubiger aber bereits gültigerweise das Fortsetzungsbegehren gestellt hat: Entweder hat der Schuldner gar nicht erst

schutz?, Diss. Zürich 2005, 211.

20 Vermerk «DV» auf dem Betreibungsregisterauszug (Art. 10 VFRR).

21 Vermerk «DB» auf dem Betreibungsregisterauszug (Art. 10 VFRR).

22 Vermerk «Z» auf dem Betreibungsregisterauszug (Art. 10 VFRR).

23 BGE 115 III 81 E. 2 
Rechtsvorschlag erhoben, oder aber der Rechtsvorschlag wurde zwischenzeitlich beseitigt. Im ersten Fall setzte sich der Schuldner nicht zur Wehr; im zweiten Fall tat er dies ohne Erfolg. Dennoch bezahlte er die offenbar geschuldete Forderung bis dahin nicht. In beiden Fällen bestehen somit Zweifel an der Zahlungswilligkeit des Schuldners. Wie es um die Zahlungsfähigkeit des Schuldners steht, kann in diesem Stadium noch nicht gesagt werden.

13 Differenzierter ist zu beurteilen, welche Schlüsse aus einer Betreibung gezogen werden können, die sich im Stadium des erhobenen Rechtsvorschlages befindet. In diesem frühen Stadium des Betreibungsverfahrens wurden von den Beteiligten erst minimale Verfahrensschritte unternommen: Der Gläubiger stellte beim Betreibungsamt das Betreibungsbegehren, das Betreibungsamt stellte hierauf den Zahlungsbefehl aus und stellte diesen dem Schuldner zu, wogegen der Schuldner Rechtsvorschlag erhob. ${ }^{24}$ Eine autoritative Feststellung, ob der Anspruch des Gläubigers besteht oder auch nur bestehen könnte, hat in diesem Zeitpunkt noch nicht stattgefunden. 25

14 Mit Sicherheit lässt sich in diesem Verfahrensstadium erst sagen, dass der Gläubiger behauptet, der Schuldner schulde ihm Geld, und der Schuldner dies bestreitet. Ob ein Rückschluss auf die Zahlungswilligkeit und Zahlungsfähigkeit des Betriebenen möglich ist, hängt davon ab, warum die Betreibung eingeleitet wurde. Die (bislang) unterlassene Beseitigung des Rechtsvorschlags ist sichtbare Folge eines unbeobachtbaren Sachverhalts, sie ist mit anderen Worten ein Indiz. Die folgende Tabelle zeigt in einem Überblick, welche Tatsachen dazu führen können, dass der Rechtsvorschlag (noch) nicht beseitigt wurde, ${ }^{26}$ und ob ein Rückschluss, zumindest probabilistischer Art, auf die Zahlungswilligkeit oder Zahlungsfähigkeit des Betriebenen möglich ist.
Art. 67-74 SchKG.

25 Abgesehen von den Fällen, in denen der Gläubiger mit der Betreibung die Vollstreckung eines gerichtlichen Urteils (oder eines Urteilssurrogats) bezweckt, er also über einen definitiven Rechtsöffnungstitel verfügt (Art. 8o SchKG).
26 Vgl. Bericht der Kommission für Rechtsfragen des Nationalrats vom 6 . November 2007 betr. Initiative Jean Studer (Fn. 5), Ziff. 3. 


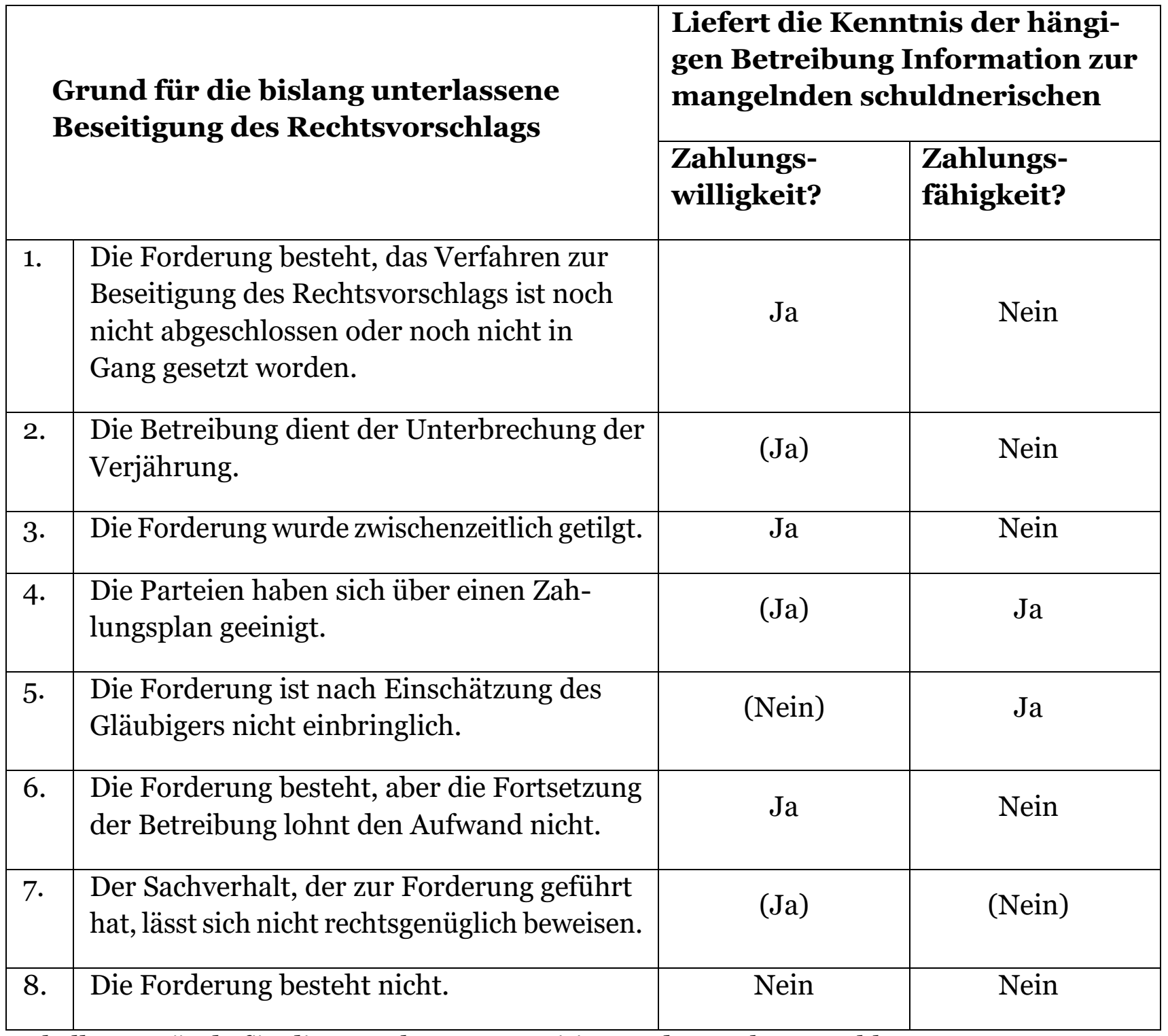

Tabelle 1: Gründe für die unterlassene Beseitigung des Rechtsvorschlags

1. Im ersten Fall gemäss obiger Tabelle hat der Gläubiger das Verfahren auf Beseitigung des Rechtsvorschlags eingeleitet oder wird es einleiten. Es ist anzunehmen, dass die Forderung tatsächlich besteht, aber auch, dass der Schuldner voraussichtlich zahlungsfähig ist, denn ansonsten würde ein rationaler, im eigenen Interesse handelnder Gläubiger die Kosten der Fortsetzung der Betreibung nicht auf sich nehmen.

Verfahren zur Beseitigung des Rechtsvorschlags können unter Um- ständen sehr lange dauern, so dass eine Fortsetzung der Betreibung selbst mehr als ein Jahr nach Zustellung des Zahlungsbefehls noch möglich ist, denn die Jahresfrist gemäss Art. 88 Abs. 2 SchKG steht während der Dauer des Verfahrens still. Die Gerichts- und Verwaltungsbehörden, bei denen ein solches Verfahren allenfalls anhängig gemacht worden ist, teilen dies dem Betreibungsamt nicht mit. Auch hat der Gläubiger nach Ablauf der Jahresfrist von Art. 88 Abs. 2 SchKG dem Betreibungsamt gegen- 
über keinen Nachweis zu erbringen, dass er ein entsprechendes Verfahren eingeleitet hat. ${ }^{27}$ Daher ist für Dritte durch Einsicht in das Betreibungsregister nicht feststellbar, ob die Fortsetzung der Betreibung noch möglich ist.

2. Im zweiten Fall dient die Betreibung dazu, die Verjährung zu unterbrechen. Der Betreibende hat keine Absicht, die Betreibung fortzusetzen, sondern will Zeit gewinnen, um die Prozessaussichten abzuklären oder eine einvernehmliche Lösung zu finden. Über die Zahlungsfähigkeit des Betriebenen vermag eine solche Betreibung nichts auszusagen (wenn überhaupt, nur Positives - der Betreibende hält es offenbar nicht für ausgeschlossen, dass der Betriebene in der Lage ist, die Forderung zu bezahlen, sonst hätte er nicht die Verjährung unterbrochen). Ob man dem Betriebenen fehlende Zahlungsbereitschaft vorwerfen kann, hängt davon $\mathrm{ab}$, ob die Forderung zu Recht besteht. Daran, respektive an der Beweisbarkeit der anspruchsbegründenden Tatsachen, hat offenbar auch der Betreibende Zweifel, sonst hätte er direkt Klage einreichen können.

3. Es kann auch sein, dass der Rechtsvorschlag nicht beseitigt wird, weil der Schuldner die Forderung zwischenzeitlich beglichen hat. Hat der Schuldner direkt an den Gläubiger bezahlt, und zieht dieser in der Folge

27 Vgl. BGE 128 III 334 (= Pra 91 [2002] Nr. 195): Der Schuldner kann nicht vom Betreibungsamt verlangen, dass es dem Gläubiger Frist ansetze, eine Anerkennungsklage einzuleiten oder ein Rechtsöffnungsbegehren zu stellen, unter der Androhung, im Säumnisfall gelte die Betreibung als zurückgezogen. die Betreibung nicht zurück, so bleibt die Betreibung im Stadium des erhobenen Rechtsvorschlags im Betreibungsregister verzeichnet. ${ }^{28}$ In diesem Fall belegt der Betreibungsregistereintrag tatsächlich eine fehlende Zahlungswilligkeit des Schuldners der Schuldner bezahlte schliesslich erst unter Betreibungsdruck -, doch nicht dessen fehlende Zahlungsfähigkeit, denn zahlen konnte er offensichtlich.

4. Weiter kann es sein, dass sich die Parteien über einen Zahlungsplan geeinigt haben, und der Gläubiger seine Betreibung nicht zurückgezogen hat. Solange der Zahlungsplan vom Schuldner eingehalten wird, hat der Gläubiger keinen Grund, Schritte zur Weiterführung der Betreibung einzuleiten. Hier zeigt der Betreibungsregistereintrag zumindest eine fehlende Zahlungsfähigkeit an; möglicherweise auch eine fehlende Zahlungswilligkeit, denn der Schuldner hätte auch ohne Einleitung eines Betreibungsverfahrens von sich aus auf den Gläubiger zugehen und ihm einen Zahlungsplan vorschlagen können.

5. Es kann auch sein, dass der Gläubiger zum Schluss kommt, dass die Forderung mutmasslich nicht einbringlich sein wird. Dies ist eine für Dritte wertvolle Information: Zwar kann es sein, dass sich der Gläubiger täuscht. Da er ein eigenes Interesse daran hat, dass seine Beurteilung richtig ist, wird er die Beurteilung aber nach bestem Wissen und Gewissen treffen. Eine solche Beurteilung ist zumindest

28 BGer, Urteil 7B.224/2006 E. 2.2.4 vom 22. Februar 2007; BGE 128 III 334, 336 (= Pra 91 [2002] Nr. 195). 
ein Indiz dafür, dass der Schuldner nicht zahlungsfähig ist. $\mathrm{Ob}$ der Schuldner zahlungswillig wäre, lässt sich nicht sagen - wenn er tatsächlich zahlungsunfähig ist, kann er auch beim besten Willen nicht zahlen.

6. Davon zu unterscheiden ist der Fall, in dem der Gläubiger zum Schluss kommt, dass die ungedeckten Kosten der Rechtsdurchsetzung den Wert der Forderung übersteigen. Es ist ein offenes Geheimnis, dass die in Zivilverfahren zugesprochenen Parteientschädigungen bei geringem Streitwert die tatsächlichen Anwaltskosten nicht decken. Ist der Forderungsbetrag daher relativ gering, der Nachweis des Bestands der Forderung aber aufwendig, wird ein rational handelnder Gläubiger unter Umständen auf die Durchsetzung auch einer bestehenden Forderung verzichten. In diesem Fall fehlt es zumindest an der Zahlungswilligkeit des Schuldners; ob er zahlungsfähig wäre, lässt sich nicht sagen.

7. Ein Grenzfall liegt vor, wenn die Forderung zwar tatsächlich besteht, sich der Sachverhalt, der zu ihrer Entstehung geführt hat - z.B. ein fahrlässiges Tun oder Unterlassen des Schuldners - aber nicht rechtsgenüglich beweisen lässt. Ob der Schuldner zahlungsfähig ist, weiss man nicht. Zahlungswillig ist er offensichtlich nicht, aber die Frage ist, ob man von einem Schuldner erwarten kann, eine Forderung zu bezahlen, von der er weiss, dass sie zwar besteht, aber nicht durchsetzbar ist. Die Autoren geben offen zu, dass sie sich in dieser Frage nicht einigen konnten.
8. Schliesslich kann es vorkommen, dass die betriebene Forderung nicht existiert und der Betreibende dies weiss oder wissen müsste. Solche Betreibungen werden hier als «ungerechtfertigt» bezeichnet. ${ }^{29}$ Hier wird der Betreibende auf weitere Schritte verzichten, weil er weiss, dass er - unter Kostenfolgen - unterliegen wird. In diese Kategorie fallen einerseits Schikane- oder Rachebetreibungen, bei denen es dem Gläubiger von Anfang an gar nicht darum geht, eine Forderung gegen den Schuldner durchzusetzen, sondern vielmehr darum, den Schuldner zu schädigen, indem er dessen Kreditansehen durch den Betreibungsregistereintrag beeinträchtigt. Andererseits fallen darunter auch «erpresserische» Betreibungen, bei denen der Betreibende hofft, dass der Betriebene im Gegenzug für den Rückzug der Betreibung - zur Vermeidung eines für Dritte während fünf Jahren sichtbaren Betreibungsregistereintrags - eine nicht bestehende Forderung bezahlt. In beiden Fällen ist der Betreibungsregistereintrag offensichtlich weder ein Indiz für die fehlende Zahlungswilligkeit noch für die fehlende Zahlungsfähigkeit des Betriebenen und sollte nicht an Dritte bekannt gegeben werden. ${ }^{30}$

15 Strittig ist, wie oft Betreibungen von nicht bestehenden Forderungen vorkommen. Da der Gläubiger die Kosten des Zahlungsbefehls vorzuschiessen

$29 \overline{\text { Die Terminologie ist nicht einheitlich, siehe Jean- }}$ Daniel Schmid, Der Ausschluss des Einsichtsrechts in das Betreibungsregister gemäss Art. 8b VE-SchKG, Jusletter 7. Oktober 2013, Rz. 13.

3o Iqbal (Fn. 19), 208 ff., kommt aufgrund einer grundrechtskonformen Auslegung zum gleichen Ergebnis. 
hat, ${ }^{31}$ müssen seine Interessen an der Betreibung einer nicht existierenden Forderung zumindest höher als die (allerdings relativ geringen) Kosten des Zahlungsbefehls sein. Die Rechtsprechung geht, jedoch ohne empirische Grundlage, davon aus, dass Betreibungen ohne Grund selten vorkommen. ${ }^{32}$ Stimmen in der Literatur meinen, dass ungerechtfertigte Betreibungen «gar nicht so selten» 33 vorkommen bzw. sogar «alltäglich» 34 seien. 35 Die Kommission für Rechtsfragen des Nationalrats geht davon aus, dass «reine Schikanebetreibungen» äusserst selten seien. ${ }^{6}$ Eine empirische Grundlage fehlt allen diesen Äusserungen. ${ }^{37}$ Das dürfte auch auf absehbare Zeit so bleiben, weil eine Klärung, ob der Betreibung eine Forderung zugrunde liegt, ohne kontradiktorisches Verfahren mit Beweiserhebung kaum denkbar ist.

\section{Möglichkeiten zur Aufhebung un- gerechtfertigter Betreibungen de lege lata}

\section{1. Überblick}

16 Grundlage für die Beschränkung des Einsichtsrechts Dritter in das Betreibungsregister ist Art. 8a Abs. 3 lit. a SchKG: Nach dieser Bestimmung geben die Betreibungsämter Dritten von einer Betrei-

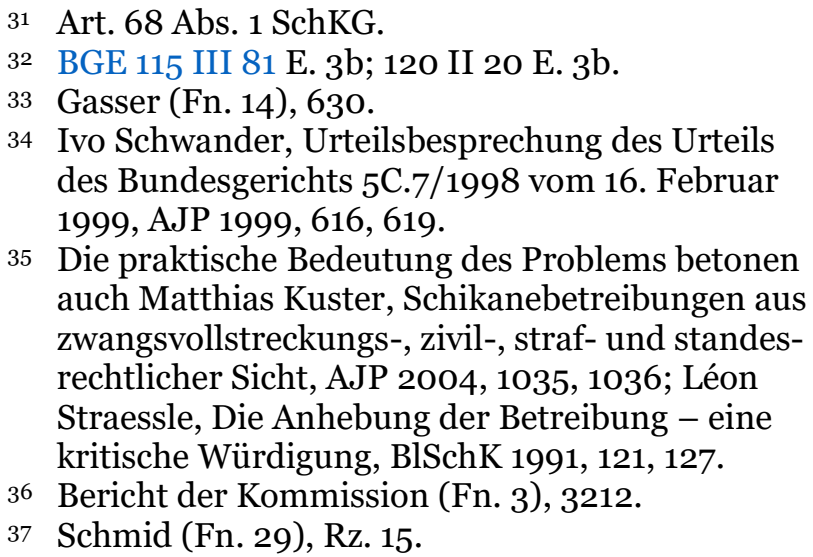

35 Die praktische Bedeutung des Problems betonen auch Matthias Kuster, Schikanebetreibungen aus zwangsvollstreckungs-, zivil-, straf- und standesrechtlicher Sicht, AJP 2004, 1035, 1036; Léon Straessle, Die Anhebung der Betreibung - eine kritische Würdigung, BlSchK 1991, 121, 127.

36 Bericht der Kommission (Fn. 3), 3212.

7 Schmid (Fn. 29), Rz. 15.

bung keine Kenntnis, wenn die Betreibung nichtig ist oder aufgrund einer Beschwerde oder eines gerichtlichen Urteils aufgehoben worden ist. Im Folgenden wird aufgezeigt, welche Wege ein Schuldner einschlagen kann, wenn er die Aufhebung einer ungerechtfertigten Betreibung bewirken will, und welche Vorund Nachteile mit den einzelnen Instrumenten verbunden sind.

\section{Klage auf Aufhebung oder Einstel- lung der Betreibung im ordentli- chen oder vereinfachten Verfahren nach Art. 85a SchKG}

17 Gemäss Art. 85a Abs. 1 SchKG kann der Betriebene jederzeit vom Gericht des Betreibungsortes feststellen lassen, dass die Schuld nicht oder nicht mehr besteht oder gestundet ist. Im Falle der Klagegutheissung hebt das Gericht die Betreibung auf oder stellt sie ein (Art. 85a Abs. 3 SchKG). Diese Klage entfaltet sowohl materiell- als auch betreibungsrechtliche Wirkungen. $3^{8}$ Ihre Gutheissung führt materiellrechtlich zur gerichtlichen Feststellung, dass die Forderung nicht oder nicht mehr besteht bzw. noch nicht fällig ist. Ihre Abweisung hat zur Folge, dass festgestellt wird, dass die betreffende Forderung besteht bzw. fällig ist.39 In betreibungsrechtlicher Hinsicht führt die Gutheissung der Klage zur Aufhebung oder Einstellung der Betreibung. ${ }^{40}$

Nicht jede Gutheissung der Klage führt ohne weiteres zum Ausschluss des Ein-

\footnotetext{
38 Statt vieler BGE 132 III 89 E. 1.1.

39 Bernhard Bodmer/Jan Bangert, in: Adrian Staehelin/Thomas Bauer/Daniel Staehelin (Hrsg.), Basler Kommentar, Bundesgesetz über Schuldbetreibung und Konkurs I, Art. 1-158 SchKG, 2. Aufl., Basel 2010 (nachfolgend «BSKSchKG-Bearbeiter»), Art. 85a N 31, m.w.H.

40 BGE 132 III 89 E. 1.1.
} 
sichtsrechts: Nach der Rechtsprechung des Bundesgerichts wird hierfür vorausgesetzt, dass die Betreibung bereits bei ihrer Einleitung ungerechtfertigt war, die Forderung somit bereits in diesem Zeitpunkt nicht (mehr) bestanden hat. ${ }^{11}$ Vor dem Hintergrund des Informationswerts des Betreibungsregisters ist dieser Rechtsprechung zuzustimmen.

19 Entgegen dem Wortlaut von Art. 85a Abs. 1 SchKG («jederzeit») kann die Klage gemäss bundesgerichtlicher Rechtsprechung nur während laufender Betreibung anhängig gemacht werden:42 Das Bundesgericht verlangt als Eintretensvoraussetzung, dass der Zahlungsbefehl rechtskräftig geworden ist, der Schuldner somit keinen Rechtsvorschlag erhoben hat oder dieser beseitigt oder zurückgezogen worden ist.43 Trotz heftiger Kritik in der Lehre, die insbesondere auf das Interesse des Betriebenen verweist, das Einsichtsrecht Dritter möglichst bald mittels Aufhebung der Betreibung auszuschliessen,44 hält das Bundesgericht an dieser Rechtsprechung fest (nach der neusten Rechtsprechung steht dem Betriebenen allerdings immer die allgemeine negative Feststellungsklage offen, siehe gleich anschliessend Ziff. 3.). 45 Der Nachweis eines besonderen

BGE 125 III 334 E. 3.

42 BGE 127 III 41 E. 4c.

43 BGE 125 III 149.

44 Barbara Graham-Siegenthaler/Marc Bernheim, Die bundesgerichtliche Rechtsprechung zur negativen Feststellungsklage gemäss Art. 85a SchKG eine kritische Würdigung, SJZ 96 (2000), 177, 179 ff.; Kuster (Fn. 35),1037; Luca Tenchio, Feststellungsklagen und Feststellungsprozess nach Art. 85a SchKG, Diss. Zürich 1999, 71 ff.; Schwander (Fn. 34), 617 ff.; Karl Spühler/Luca Tenchio, Feststellungsklagen gemäss Art. 85a Abs. 1 SchKG nach gültig erhobenem Rechtsvorschlag?, AJP 1999, 1241, 1245 ff.

45 BGE 128 III 334; BGE 132 III 277 E. 4.2 (= Pra 96 [2007] Nr. 10); BGer, Urteil 4C.2/2006 vom 21.
Feststellungsinteresses ist bei der Klage nach Art. 85a SchKG nicht erforderlich. 46

20 Die Klage wird gutgeheissen, wenn die in Betreibung gesetzte Forderung nicht $\mathrm{o}-$ der nicht mehr besteht oder gestundet ist, wobei der Nichtbestand der Forderung vom Kläger (Schuldner) nur behauptet werden muss.47 Die Behauptungs-, Substantiierungs- und Beweislast für die forderungsbegründenden Tatsachen trägt der Beklagte (Gläubiger). 48 Gemäss der üblichen Beweislastverteilung trägt der Kläger hingegen die Beweislast für die rechtsaufhebenden bzw. vernichtenden und rechtshindernden Tatsachen. 49

21 Hauptnachteil der Klage nach Art. 85a SchKG ist - neben der Dauer und der Kosten, insbesondere der Kautionierung der Gerichtskosten ${ }^{0}$ - dass sie nur bei nicht erhobenem oder beseitigtem Rechtsvorschlag zulässig ist. In all den Fällen, in denen der Betreibende die Betreibung nach erhobenem Rechtsvorschlag nicht weiter verfolgt, ist die Klage nach Art. 85a SchKG daher kein taugliches Mittel für den Betriebenen, sich gegen den unliebsamen Betreibungsregistereintrag zur Wehr zu setzen. Man wird dem Betriebenen kaum raten können,

März 2006, E. 3.4; zustimmend Reto Vonzun/André Equey, Mittel und Wege zur Beseitigung der negativen Auswirkungen des Betreibungsregistereintrags grundloser Betreibungen, AJP 2011, 1337, 1341.

46 BGE 129 III 197 E. 2.5 unter Hinweis auf Spühler/Tenchio (Fn. 44), 1241 f.; BSK-SchKGBodmer/Bangert (Fn. 39), Art 85a N 4.

47 Tenchio (Fn.44), 146.

48 BSK-SchKG-Bodmer/Bangert (Fn. 39), Art. 85a N 4; Tenchio (Fn. 44), 146; a.M. Karl Spühler, Schuldbetreibungs- und Konkursrecht I, 5. A., Zürich/Basel/Genf 2010, N 725.

49 Hans Schmid, Negative Feststellungsklagen, AJP 2002, 774, 781 Fn. 66.

50 Art. $98 \mathrm{ZPO}$. 
auf die Erhebung des Rechtsvorschlags $\mathrm{zu}$ verzichten, um sich die Möglichkeit einer Klage nach Art. 85a SchKG offen zu halten. ${ }^{1}$ Art. $85 \mathrm{a}$ Abs. 1 E-SchkG sieht denn hier auch eine Korrektur vor; gemäss dem Gesetzesvorschlag soll die Klage nach Art. 85a «ungeachtet eines allfälligen Rechtsvorschlages» jederzeit eingereicht werden können.

\section{Allgemeine negative Feststellungs- klage}

Die allgemeine negative Feststellungsklage ${ }^{2}$ steht dem Betriebenen im Gegensatz zur Klage nach Art. 85a SchKG auch dann zur Verfügung, wenn die Betreibung infolge Rechtsvorschlags zum Stillstand gekommen ist.53 Mit der allgemeinen Feststellungsklage kann der Betriebene die gerichtliche Feststellung des Nichtbestehens der in Betreibung gesetzten Forderung (mit voller materieller Rechtskraft) und der Grundlosigkeit der Betreibung beantragen, d.h. das Nichtbestehen der Schuld auch im Zeitpunkt der Einleitung der Betreibung.54 Letzteres ist für den Ausschluss des Einsichtsrechts Dritter nach der bundesgerichtlichen Rechtsprechung erforderlich.55 Aus Sicht des Informationswerts des Betreibungs-

51 Der Betriebene, der bewusst keinen Rechtsvorschlag erhebt oder den zuvor erhobenen Rechtsvorschlag wieder zurückzieht, um sich so die Klagemöglichkeit von Art. 85a SchKG zu verschaffen, muss sich unter Umständen ein rechtsmissbräuchliches Verhalten vorwerfen lassen, mit der Folge, dass auf seine Klage nicht eingetreten wird (so geschehen in ZR 109 [2010] Nr. 6). Das Obergericht des Kantons Zürich verneinte indessen (unseres Erachtens zu Recht) das Vorliegen eines offenbaren Rechtsmissbrauchs in einer solchen Konstellation (ZR 109 [2010] Nr. 69).

52 Art. 88 ZPO.

53 BGE 132 III 277 E. 4.2; Vonzun/Equey (Fn. 45), 1341.

54 BGE 120 II 20 E. 2 und E. 3c.

55 BGE 125 III 334 E. 3; Vonzun/Equey (Fn. 45), 1343 . registers ist dieser Rechtsprechung zuzustimmen, denn wird eine bestehende Forderung in Betreibung gesetzt und geht sie erst später unter, so lässt dies Rückschlüsse auf die (fehlende) Zahlungswilligkeit des Betriebenen zu.

23 Bis vor kurzem war jedoch nach der bundesgerichtlichen Rechtsprechung das Rechtsschutzinteresse des Betriebenen an einer negativen Feststellungsklage nur dann zu bejahen, wenn namhafte Beträge und nicht bloss Bagatellbeträge in Betreibung gesetzt wurden und wenn der Betriebene dartun konnte, dass er konkret aufgrund der Betreibung in seiner wirtschaftlichen Bewegungsfreiheit behindert wird. Dem Gläubiger blieb zudem der Nachweis offen, dass ihm die Beweisführung gegenwärtig aus triftigen Gründen nicht zuzumuten ist. ${ }^{6}$ In einem kürzlich ergangenen Urteil trug das Bundesgericht der an der bisherigen Rechtsprechung geäusserten Kritik Rechnung und lockerte diese Praxis. Es hielt fest, dass das schutzwürdige Interesse an der Feststellung des Nichtbestands der Forderung grundsätzlich zu bejahen ist, sobald diese in Betreibung gesetzt wurde, ohne dass der Feststellungskläger konkret nachweisen muss, dass er wegen der Betreibung in seiner wirtschaftlichen Bewegungsfreiheit empfindlich beeinträchtigt wird. Vorbehalten wurde jedoch der Fall, in dem die Betreibung nachweislich einzig zur Unterbrechung der Verjährung eingeleitet wurde, nachdem der (angebliche) Schuldner die Unterzeichnung einer Verjährungsverzichtserklärung verweigert hat, und die Forderung vom (angeblichen) Gläubiger aus triftigen Gründen nicht sofort im vollen

56 BGE 120 II 20 E. 3 b. 
Umfang geltend gemacht werden kann. 57

Diese Praxisänderung ist zu begrüssen. Sie trägt dem Umstand Rechnung, dass auch Betreibungen über bloss geringfügige Beträge die Gefahr begründen, dass Dritte an der Kredit- und Vertrauenswürdigkeit des Betriebenen zweifeln. ${ }^{8}$

Nicht gerechtfertigt scheint unseres Erachtens aber der vom Bundesgericht angebrachte Vorbehalt für den Fall, dass die Betreibung einzig zur Unterbrechung der Verjährung eingeleitet wurde: Für das Unterbrechen der Verjährung ist nicht erforderlich, dass der (angebliche) Schuldner auf Dauer hin mit einem Betreibungsregistereintrag belastet ist. Die Unterbrechungswirkung tritt bereits mit der Postaufgabe des Betreibungsbegehrens ein, und sogar auch dann, wenn der Betreibende dem Betreibungsamt umgehend (u.U. gleichzeitig mit dem Stellen des Betreibungsbegehrens) den Rückzug der Betreibung mitteilt.59 Es leuchtet deshalb nicht ein, weshalb dem Betriebenen die Möglichkeit einer negativen Feststellungsklage verwehrt bleiben soll, wenn der Gläubiger die Betreibung (einzig) zum Zwecke der Verjährungsunterbrechung eingeleitet hat und sich weigert, die Betreibung zurückzuziehen.

Vorteil der allgemeinen negativen Feststellungsklage gegenüber der Klage nach Art. 85a SchKG ist demnach, dass sie auch im Stadium des erhobenen Rechts-

57 BGer, Urteil 4A_414/2014 vom 16. Januar 2015, E. 2.7 (zur Publikation vorgesehen).

58 Vonzun/Equey (Fn. 45), 1345.

59 Robert K. Däppen, in Heinrich Honsell/Nedim Peter Vogt/Wolfgang Wiegand, Basler Kommentar, Obligationenrecht I, 5. Aufl., Basel 2011 (nachfolgend «BSK-OR-Bearbeiter»), Art. $135 \mathrm{~N}$ 6; BSK-SchKG-Kofmel Ehrenzeller (Fn. 39), Art. $68 \mathrm{~N} 48$. vorschlags zulässig ist. Nachteilig wirkt sich für den Betriebenen jedoch aus, dass er die Gerichtskosten vorzuschiessen hat, wobei sich der Streitwert nach der Höhe der Betreibungsforderung richtet. Gerade dann, wenn es sich um eine Schikanebetreibung in exorbitanter Höhe handelt, kann dies für den Betriebenen eine prohibitive Wirkung haben. Selbst im Erfolgsfall erhält er nur eine Regressforderung gegen den Betreibenden.60 Ist der Betreibende zahlungsunfähig, bleibt der Betriebene letztlich auf den Kosten sitzen.

\section{Klage auf Aufhebung oder Einstel- lung der Betreibung im summari- schen Verfahren nach Art. 85 SchKG}

27 Gemäss Art. 85 SchKG kann der Betriebene jederzeit beim Gericht des Betreibungsortes im summarischen Verfahren die Aufhebung bzw. die Einstellung der Betreibung verlangen, wenn er durch Urkunden beweist, dass die Schuld samt Zinsen und Kosten getilgt oder gestundet ist. Die Klage nach Art. 85 SchKG ist rein betreibungsrechtlicher Natur; sie hat ausschliesslich betreibungsrechtliche Wirkungen im Rahmen der betreffenden Betreibung. ${ }^{61}$ Der materielle Bestand der in Betreibung gesetzten Forderung ist nur eine Vorfrage. ${ }^{62}$ Entsprechend erlangt das Urteil für die in Betreibung gesetzte Forderung keine materielle Rechtskraft. ${ }^{63}$

60 Art. 111 Abs. 2 ZPO

61 BGer, Urteil 5P.328/2006 vom 1. Februar 2007, E. 1.2; Urteil 5P.8/2005 vom 3. Mai 2005, E. 1.1; BSK-SchKG-Bodmer/Bangert (Fn. 39), Art. 85 N 35 .

62 Kurt Amonn/Fridolin Walther, Grundriss des Schuldbetreibungs- und Konkursrechts, 9. Aufl., Bern 2013, § $20 \mathrm{~N} 5$.

63 BGE 125 III 149 E. 2b.aa. 

85 SchKG führt zumindest dann, wenn die Forderung bereits bei Anhebung der Betreibung nicht bestanden hat, dazu, dass Dritte von der Betreibung keine Kenntnis mehr erhalten. ${ }^{64}$

Art. 85 SchKG setzt eine hängige Betreibung, nach der neueren Rechtsprechung des Bundesgerichts (BGE 140 III 41) jedoch anders als die Klage nach Art. 85a SchKG keinen rechtskräftigen Zahlungsbefehl, voraus. 65 Das Bundesgericht begründet dies mit der fehlenden materiellen Rechtskraft des Urteils im Verfahren nach Art. 85 SchKG: weil es sich um eine rein betreibungsrechtliche Klage handelt, steht es dem Gläubiger offen, im Fall seines Unterliegens mit der Forderungsklage gegen den Schuldner vorzugehen. Daher sei das Interesse des Gläubigers, nicht vorzeitig den Beweis seiner Forderung antreten $\mathrm{zu}$ müssen, viel weniger berührt. 66

Obwohl das Bundesgericht die Frage in BGE 140 III 41 nicht ausdrücklich beantwortet, bedarf es für die Zulässigkeit der Klage nach Art. 85 keines weiteren Interesses als dasjenige, Dritten die Einsicht in die aufgehobene Betreibung zu verweigern. ${ }^{67}$ Dies gilt auch dann, wenn der Kläger einem vorgängigen Ersuchen des Beklagten um Abgabe einer Verjährungsverzichtserklärung nicht nachgekommen ist und dieser die Betreibung angeblich zur Verjährungsunterbrechung eingeleitet hat. 68

Botschaft SchKG (Fn. 6), 32; Vonzun/Equey (Fn. 45), 1346.

65 BGE 140 III 41 E. 3.2.3.

66 BGE 140 III 41 E. 3.2.3.

67 BSK-SchKG-Bodmer/Bangert (Fn. 39), Art. 85 N 4 und 12; Vonzun/Equey (Fn. 45), 1350.

68 Vonzun/Equey (Fn. 45), 1350.
31 Nach dem Wortlaut spricht Art. 85 SchKG nur von Tilgung und Stundung der Schuld, nicht aber von deren Nichtbestand. Das Bundesgericht lässt aber auch den Nachweis des Nichtbestehens der Schuld (im Zeitpunkt der Einleitung der Betreibung) genügen.69

32 Wichtigste Einschränkung der Klage nach Art. 85 SchKG gegenüber der Klage nach Art. 85a SchKG und der allgemeinen negativen Feststellungsklage ist die Beschränkung der zulässigen Beweismittel auf Urkunden: Den Nachweis der Tilgung, Stundung oder des Nichtbestehens der Betreibungsforderung kann der Schuldner nur durch strikten Urkundenbeweis erbringen; die blosse Glaubhaftmachung ist nicht ausreichend. 70

33 Damit ist auch der Hauptnachteil der Klage nach Art. 85 SchKG angesprochen. Während Tilgung meist durch Urkunden (Quittung) belegt werden kann, wird eine Stundung oft auch mündlich erfolgen und im Verfahren nach Art. 85 SchKG nicht nachzuweisen sein. Den Beweis des Nichtbestehens einer Forderung mittels Urkunden zu führen, dürfte nur in den seltensten Fällen gelingen. ${ }^{71}$ Zur Löschung ungerechtfertigter Betreibungen aus dem Betreibungsregister ist die Klage nach Art. 85 SchKG daher wenig geeignet.

69 BGE 140 III 41 E. 3.3.1.

70 BGE 140 III 41 E. 3.3.2; 125 III 149 E. 2b/aa.

71 Er ist dem Kläger in BGE 140 III 41 denn auch nicht gelungen, siehe dort, E. 3.4.1. 
5. Geltendmachung der Nichtigkeit der Betreibung mittels betreibungsrechtlicher Beschwerde nach Art. 17 SchKG

34 Wenn offensichtlich ist, dass der Gläubiger mit der Betreibung Ziele verfolgt, die mit der Zwangsvollstreckung nicht das Geringste zu tun haben, so ist die Betreibung rechtsmissbräuchlich (Art. 2 Abs. 2 ZGB) und damit nichtig. $7^{2}$ Die Nichtigkeit der Betreibung ist jedoch nicht leichthin anzunehmen. Solange der Gläubiger mit der Betreibung zumindest versucht, einen Anspruch durchzusetzen - mag dieser auch zweifelhaft sein - erscheint ein Rechtsmissbrauch nahezu ausgeschlossen, denn der Gläubiger verwendet das Institut der Zwangsvollstreckung in diesem Fall nicht zweckwidrig. Ein rechtsmissbräuchliches Vorgehen des Gläubigers kann angenommen werden, wenn der Betreibung einzig zwangsvollstreckungsfremde Motive zu Grunde liegen, wie etwa Kreditschädigung, Verwirrung, Bedrängung, Zermürbung oder Schikanierung des Schuldners.73 In einem Urteil von September 2014 betrachtete das Bundesgericht eine Betreibung als rechtmissbräuchlich, weil die gleiche Forderung bereits in Betreibung gesetzt worden war und die Parteien Vergleichsgespräche führten. Unter den Umständen verstosse eine erneute Betreibung für die gleiche Forderung gegen das Verbot widersprüchlichen Verhaltens. 74

Liegt ein rechtsmissbräuchliches Vorgehen des Gläubigers vor, so ist das Betreibungsamt befugt und verpflichtet, die

72 BSK-SchKG-Wüthrich/Schoch (Fn. 39), Art. 69 N 16.

73 BSK-SchKG-Wüthrich/Schoch (Fn. 39), Art. 69 $\mathrm{N} 16$.

74 BGer, Urteil 5A_508/2014 vom 19. September 2014, E. 2.3.2, 2.3.3.
Nichtigkeit der Betreibung festzustellen und die Ausstellung des Zahlungsbefehls zu verweigern. $75 \mathrm{Ob}$ jedoch der Gläubiger mit seiner Betreibung tatsächlich eine Forderung zwangsvollstreckungsrechtlich durchsetzen will, oder ob er mit der Betreibung ausschliesslich sachfremde Ziele verfolgt, dürfte für das Betreibungsamt anhand der Angaben im Betreibungsbegehren nur in den seltensten Fällen je ersichtlich sein. ${ }^{6} \mathrm{Im}$ Zweifel hat das Betreibungsamt den Zahlungsbefehl auszustellen und das Betreibungsverfahren in Gang zu setzen. 77

36 Sieht sich der Schuldner mit einer Betreibung konfrontiert, die in seinen Augen nicht nur ungerechtfertigt, sondern rechtsmissbräuchlich ist, so kann er gegen den Zahlungsbefehl Beschwerde im Sinne von Art. 17 SchKG führen. Im Beschwerdeverfahren kann der Schuldner die Nichtigkeit des Zahlungsbefehls und der Betreibung an sich feststellen lassen. ${ }^{8}$ Da die Nichtigkeit von behördlichen Verfügungen ohnehin jederzeit geltend gemacht werden kann und von Amtes wegen zu beachten ist, 79 kann sich der Schuldner auch ausserhalb einer gegen den Zahlungsbefehl gerichteten Beschwerde auf die Nichtigkeit der Betreibung berufen. Dies ist insbesondere dann relevant, wenn die zehntägige Beschwerdefrist $^{80}$ für eine Beschwerde gegen den Zahlungsbefehl bereits abgelaufen ist.

75 BSK-SchKG-Wüthrich/Schoch (Fn. 39), Art. 69 $\mathrm{N} 16$.

76 BGer, Urteil 5A_508/2014 vom 19. September 2014, E. 2.4.

77 Vonzun/Equey (Fn. 45), 1339.

78 Vonzun/Equey (Fn. 45), 1339, m.w.H.

79 BSK-SchKG-Cometta/Möckli (Fn. 39), Art. 22 $\mathrm{N} 20$.

8o Art. 17 Abs. 2 SchKG. 
37 Eine nichtige Betreibung ist vom Einsichtsrecht Dritter ausgeschlossen (Art. 8a Abs. 3 lit. a SchKG). Auch wenn im Beschwerdeverfahren der Bestand der betriebenen Forderung nicht geprüft wird, ${ }^{81}$ ist die Feststellung der Nichtigkeit einer Betreibung wegen Rechtsmissbrauchs ein starkes Indiz dafür, dass die betriebene Forderung nicht existiert. Der Ausschluss des Einsichtsrechts ist aus Sicht des Informationswerts des Betreibungsregisters daher erwünscht.

Die Geltendmachung der Nichtigkeit im Beschwerdeverfahren ist für den betroffenen Schuldner verfahrenstechnisch vorteilhaft, da das Beschwerdeverfahren grundsätzlich kostenlos ist. ${ }^{82}$ Zudem werden im Beschwerdeverfahren auch keine Parteientschädigungen zugesprochen, 83 was das Prozessrisiko des Beschwerdeführers im Vergleich zu einem gewöhnlichen Zivilprozess erheblich mindert. Dem nicht anwaltlich vertretenen Beschwerdeführer kommt schliesslich der Umstand entgegen, dass das Beschwerdeverfahren vom Untersuchungsgrundsatz beherrscht ist, die Aufsichtsbehörden somit den Sachverhalt von Amtes wegen festzustellen haben. ${ }^{84}$ Der praktische Nachteil der Beschwerde ist, dass es nur in den seltensten Fällen gelingen wird, zwangsvollstreckungsfremde Motive nachzuweisen.

81 BGer, Urteil 5A_508/2014 vom 19. September 2014, E. 2.3.1.

82 Art. 20a Abs. 2 Ziff. 5 SchKG. Vorbehalten bleibt die bös- oder mutwillige Prozessführung.

83 Art. 62 Abs. 2 GebV SchKG.

84 Art. 20a Abs. 2 Ziff. 2 SchKG. In der Praxis darf dieser Vorteil aber nicht überschätzt werden, da die SchKG-Beschwerdebehörden über keinen Ermittlungsapparat verfügen und daher auf die Parteivorbringen angewiesen sind.

\section{Zusammenfassung}

39 Allen rechtlichen Möglichkeiten zur Aufhebung ungerechtfertigter Betreibungen, respektive Einschränkungen des Einsichtsrechts Dritter in das Betreibungsregister, nach geltendem Recht ist gemeinsam, dass sie auf einer individuellen Beurteilung des konkreten Einzelfalls mit all den damit verbundenen Vor- und Nachteilen beruhen:

40 Vorteil der individuellen Beurteilung ist, dass die Betreibung nur aufgehoben wird, wenn die Forderung tatsächlich nicht besteht (respektive im Falle der Aufhebung wegen Rechtsmissbräuchlichkeit mit grösster Wahrscheinlichkeit nicht besteht). Damit ist mit der Streichung der entsprechenden Betreibung aus dem Betreibungsregister (respektive der Einschränkung des Einsichtsrechts) immer auch eine Verbesserung des Informationswerts des Betreibungsregisters verbunden, denn Betreibungen über nicht existierende Forderungen liefern keine Informationen zur Zahlungswilligkeit und -fähigkeit des Betriebenen.

41 Nachteil der Möglichkeiten de lege lata ist der hohe Zeit- und Kostenaufwand, der mit einer individuellen Beurteilung naturgemäss verbunden ist. Ein Zivilprozess kann bis zu einem rechtskräftigen Urteil ohne weiteres zwei bis drei Jahre dauern, ganz zu schweigen davon, dass der Kläger die Gerichtskosten vorschiessen muss und er, auch wenn er obsiegt, das Risiko trägt, dass seine Ersatzforderung beim Beklagten nicht einbringlich ist. Selbst eine betreibungsrechtliche Beschwerde dauert bis zu ihrer rechtskräftigen Erledigung schnell einmal sechs Monate. Für jemanden, der auf Wohnungssuche ist, ist dies eine lange Zeit, 
während der sein Betreibungsregisterauszug nicht «sauber» ist.

Demgegenüber hätte eine schematische Beschränkung des Einsichtsrechts, wie sie der Gesetzesvorschlag der Kommission für Rechtsfragen des Nationalrats vom 19. Februar 2015 vorsieht, den Vorteil, dass sie unmittelbar wirkt und kostenfrei ist. Nachteil jeder schematischen Lösung ist aber, dass damit auch das Einsichtsrecht betreffend gerechtfertigter Betreibungen eingeschränkt wird. ${ }^{85}$ Der Informationswert des Betreibungsregisters wird durch eine schematische Lösung nur dann verbessert, wenn diese deutlich mehr ungerechtfertigte als gerechtfertigte Betreibungen erfasst. Im Folgenden sollen die derzeit diskutierten Lösungsvorschläge daraufhin untersucht werden, ob sie den Informationswert des Betreibungsregisters mutmasslich verbessern können.

\section{Der Gesetzesvorschlag der Kom- mission für Rechtsfragen des Nati- onalrats vom 19. Februar 2015}

\section{1. Überblick} von Fabio Abate verabschiedete die Kommission für Rechtsfragen des Nationalrats am 19. Februar 2015 einen Vorschlag zur Änderung des SchKG ${ }^{86}$ sowie einen Bericht ${ }^{87}$ hierzu. Eine Minderheit der Kommission beantragte, auf die Revision zu verzichten und auf die Vorlage nicht einzutreten. 88

85 Bericht der Kommission (Fn. 3), 3216.

86 Fn. 2.

87 Fn. 3.

88 Bericht der Kommission (Fn. 3), 3216.
44 Der Gesetzesvorschlag sieht vier punktuelle Änderungen des SchKG vor, welche die Stellung eines zu Unrecht Betriebenen verbessern sollen.

45 Die erste Änderung betrifft das Recht Dritter, Einsicht in das Betreibungsregister zu nehmen. ${ }^{89}$ Die vorgeschlagene Bestimmung sieht vor, dass das Betreibungsamt auf Antrag des Betriebenen Dritten von einer Betreibung, gegen die der Betriebene Rechtsvorschlag erhoben hat, vorläufig keine Kenntnis gibt, 90 ausser (i) es seien seit der Einleitung der Betreibung und in den sechs Monaten davor vor dem gleichen Betreibungsamt gegen den gleichen Betriebenen Betreibungen von mindestens zwei weiteren Gläubigern eingeleitet worden; ${ }^{91}$ oder (ii) in den letzten zwölf Monaten wurde gegen den Schuldner vor dem gleichen Betreibungsamt eine Betreibung fortgesetzt; 92 oder (iii) in den letzten zwölf Monaten wurde eine in Betreibung gesetzte Forderung durch Zahlung an das Betreibungsamt beglichen und der Gläubiger hat die Betreibung nicht zurückgezogen. 93 Diese geplante Änderung soll nachfolgend (Ziff. 2.) einer vertieften Würdigung unterzogen werden.

46 Die zweite Änderung setzt bei Art. 73 SchKG an. Nach geltendem Recht kann der Schuldner bis zum Ablauf der Frist zur Erhebung des Rechtsvorschlags vom Betreibungsamt verlangen, dass dieses den Gläubiger auffordert, die Beweismittel für seine Forderung beim Betreibungsamt zur Einsicht vorzulegen. Zweck dieser Bestimmung ist es, dem Schuldner

\footnotetext{
Art. 8b E-SchKG.

Art. 8b Abs. 1 E-SchKG.

Art. 8b Abs. 2 lit. a E-SchKG.

Art. 8b Abs. 2 lit. b E-SchKG.

Art. 8b Abs. 2 lit. c E-SchKG.
} 
den Entscheid darüber zu erleichtern, ob er die betriebene Forderung anerkennen oder bestreiten soll.94 Neu soll dem Schuldner dieses Recht während der gesamten Dauer des Betreibungsverfahrens zustehen. Zudem hat der Gläubiger nicht nur die Beweismittel betreffend die betriebene Forderung einzureichen, sondern auch eine Übersicht über alle fälligen Ansprüche gegen den Schuldner.95 Auf diese Änderung wird im vorliegenden Aufsatz nicht näher eingegangen.

Die dritte Änderung erweitert den Anwendungsbereich der Feststellungsklage nach Art. 85a SchKG. Gemäss Rechtsprechung des Bundesgerichts ist die Klage nach Art. 85a SchKG nur zulässig, wenn sie nach rechtskräftiger Beseitigung des Rechtsvorschlags (und vor Verteilung des Verwertungserlöses bzw. Konkurseröffnung) angehoben wird. ${ }^{6}$ Gemäss dem Gesetzesvorschlag soll die Klage ungeachtet eines allfälligen Rechtsvorschlags jederzeit zulässig sein. 97 Diese Änderung erscheint begrüssenswert; gleichwohl wird die Feststellungklage nach Art. 85a SchKG für den Betriebenen aufgrund des damit verbundenen Aufwands und des Kostenrisikos bloss von beschränktem Nutzen sein. $9^{8}$ Nur wenige Betriebene werden bereit sein, einen Prozess zu führen, der einem gewöhnlichen Forderungsprozess punkto Kosten- und Zeitaufwand in nichts nachsteht, nur um einen störenden Betreibungsregistereintrag löschen zu lassen.

94 BSK-SchKG-Wüthrich/Schoch (Fn. 39), Art. 73 $\mathrm{N} 1$.

95 Art. 73 Abs. 1 E-SchKG.

96 Siehe vorne, Ziff. 2.

97 Art. 85a Abs. 1 E-SchKG.

98 Bericht der Kommission (Fn. 3), 3220.
48 Die vierte und letzte Änderung betrifft schliesslich die Dauer der Fortsetzungsfrist gemäss Art. 88 SchKG. Sie soll von einem Jahr auf sechs Monate verkürzt werden. 99

\section{Würdigung von Art. 8b E-SchKG}

\section{a. Kein Systemwechsel}

49 Einleitend ist festzuhalten, dass es nach dem Gesetzesvorschlag weiterhin möglich ist, jemanden ohne Vorliegen besonderer Voraussetzungen (quasi «grundlos») zu betreiben, und im Betreibungsregister werden im Grundsatz auch weiterhin (für Dritte ersichtlich) sämtliche eingeleiteten Betreibungen aufgeführt, unabhängig davon, ob die betriebene Forderung materiell besteht. Es ist somit kein «Systemwechsel» geplant.

\section{b. Wirkung auf den vom Betriebe- nen verlangten Selbstauszug}

50 Der Gesetzesvorschlag versucht, die Stellung des Betriebenen zu verbessern, indem das Einsichtsrecht Dritter (auf Antrag) unter gewissen Voraussetzungen eingeschränkt wird. Die Ausübung des Einsichtsrechts gemäss Art. 8a SchKG ist jedoch nur eine Art, wie Dritte an Informationen des Betreibungsregisters gelangen. In der wirtschaftlichen Realität, insbesondere im Vorfeld von Vertragsabschlüssen, und insbesondere wenn der Dritte der stärkere Verhandlungspartner ist, beschafft sich der Dritte die gewünschte Informationen oftmals dadurch, dass er seinen potentiellen Vertragspartner auffordert, ihm einen Betreibungsregisterauszug betreffend die eigene Person vorzulegen. ${ }^{100}$ Hier stellt

99 Art. 88 Abs. 2 E-SchKG.

$100 \mathrm{Vgl}$. vorne, Ziff. 2. 
sich die Frage, ob der Betreibungsregisterauszug betreffend die eigene Person ebenfalls den einschränkenden Kriterien gemäss Art. 8b E-SchKG unterstünde, denn nur dann könnte der mit dem Gesetzesvorschlag verfolgte Zweck auch verwirklicht werden.

ist auszugehen. Da auch te geltende Regelung von Art. 8a Abs. 3 SchKG ausschliesslich das Einsichtsrecht Dritter erwähnt und es bereits heute einer weitverbreiteten (wenn auch uneinheitlichen Praxis) der Betreibungsämter entspricht, im Betreibungsregisterauszug betreffend die eigene Person nur diejenigen Betreibungen aufzuführen, welche auch Dritten zur Kenntnis gebracht werden dürften, ${ }^{101}$ ist anzunehmen, dass dies (wenn auch weiterhin uneinheitlich) auch unter dem neuen Art. 8b E-SchKG so gehandhabt würde. ${ }^{102}$

Der Zweck von Art. 8b E-SchKG würde allerdings dann vereitelt, wenn die Betreibungsämter auf dem Auszug vermerken würden, ob es sich beim erstellten Auszug um einen «vollständigen» Auszug handelt oder ob ein Antrag des Betriebenen auf Nichtbekanntgabe gemäss Art. 8b Abs. 1 E-SchKG vorliegt. Unseres Erachtens würde es jedoch eine zweckkonforme Auslegung von Art. 8b Abs. 1 E-SchKG den Betreibungsämtern verbieten, den Betreibungsregisterauszug entsprechend zu kennzeichnen. ${ }^{103}$

101 Schmid (Fn. 29) Rz. 17 und Fn. 89.

102 Die von Schmid (Fn. 29) in Rz. 18 geäusserten Bedenken teilen wir daher nicht.

103 Im Ergebnis gl. M. Schmid (Fn. 29) Fn. 95, zumindest was den auf Verlangen von Dritten erstellten Betreibungsregisterauszug betrifft.

\section{c. Würdigung der von Art. 8b Abs. 2 E-SchKG aufgestellten Kriterien}

53 Die Kommission für Rechtsfragen des Nationalrats geht von der unseres Erachtens zutreffenden Annahme aus, dass ein Eintrag im Betreibungsregister von Dritten durchwegs als Zeichen fehlender Zahlungsfähigkeit und Zahlungswilligkeit interpretiert wird und für den Betroffenen nachteilige Folgen haben kann. Basiert der Eintrag auf einer «ungerechtfertigten» Betreibung, so entsteht ein Bild zumindest in den Augen der meisten Dritten -, das nicht der Realität entspricht. Ziel des Gesetzesvorschlags ist es daher zu verhindern, dass ungerechtfertigte Betreibungen Dritten zur Kenntnis gebracht werden. ${ }^{104}$ Die vorgeschlagene Regelung hat sich deshalb daran zu messen, ob durch sie der Informationsgehalt des Betreibungsregisters tatsächlich verbessert wird.

54 Richtig erscheint uns, dass es nicht Aufgabe des Betreibungsamts sein kann, den materiellen Bestand der in Betreibung gesetzten Forderung zu überprüfen.105 Soweit der Entscheid, ob eine Betreibung Dritten zur Kenntnis gebracht werden darf, vom Betreibungsamt zu fällen ist, erscheint der Ansatz richtig, dass für diese Beurteilung auf einfach handzuhabende, formale Kriterien abgestellt werden soll. ${ }^{106}$ Mit dem Entscheid für eine schematische Lösung erscheint aber auch bereits klar, dass in Kauf genommen werden muss, dass nicht jede einzelne Betreibung «korrekt» vom Schema erfasst wird. Mit anderen Worten muss damit gerechnet werden, dass die Anwendung der im Gesetz definierten Kriterien dazu

\footnotetext{
104 Vgl. Bericht der Kommission, 3214.

105 Bericht der Kommission (Fn. 3), 3217.

106 Bericht der Kommission (Fn. 3), 3217.
} 
führen kann, dass eine Betreibung auch dann Dritten zur Kenntnis gebracht wird, wenn die betriebene Forderung nicht besteht, und dass umgekehrt eine Betreibung Dritten nicht zur Kenntnis gebracht wird, obwohl die Betreibung zu Recht eingeleitet wurde. ${ }^{107}$

erwähnt, sieht der Gesetzesvorschlag vor, dass eine Betreibung auf Gesuch des Betriebenen hin Dritten nicht mitgeteilt wird, sofern beim gleichen Betreibungsamt in den sechs Monaten vor Einleitung der Betreibung Betreibungen von höchstens einem weiteren Gläubiger anhängig gemacht worden sind und wenn in den letzten zwölf Monaten weder eine Betreibung fortgesetzt noch eine betriebene Forderung durch Zahlung ans Betreibungsamt beglichen wurde. Der Vorschlag geht dabei von der Überlegung aus, dass ungerechtfertigte Betreibungen typischerweise als Einzelfälle auftreten, es somit unwahrscheinlich ist, dass jemand von verschiedenen Personen ungerechtfertigt betrieben wird. ${ }^{108}$ Mit anderen Worten spricht bei Vorliegen mehrerer Betreibungen einiges dafür, dass sich unter den mehreren Betreibungen zumindest auch gerechtfertigte finden. Der vom Betreibungsregister vermittelte Eindruck der mangelhaften Zahlungsfähigkeit und Zahlungswilligkeit erscheint in diesem Fall zutreffend, selbst dann, wenn nicht jede einzelne Betreibung gerechtfertigt ist. Die Gefahr, dass sich mehrere Personen zusammentun, um je in eigenem Namen ungerechtfertigte Betreibungen gegen einen Dritten einzuleiten, scheint uns eher theoretischer Natur. ${ }^{109}$

107 Bericht der Kommission (Fn. 3), 3216; Schmid (Fn. 29) Rz. 22.

${ }_{108}$ Bericht der Kommission (Fn. 3), 3217.

109 Einige Stimmen hatten diese Gefahr gesehen, Vernehmlassungsbericht (Fn. 4), 4.
56 Verfolgt man diesen Ansatz, so würde es unseres Erachtens aber bereits genügen, wenn in den letzten sechs Monaten bereits eine Betreibung durch eine andere Person eingeleitet wurde, damit es überwiegend wahrscheinlich ist, dass es sich bei der zweiten Betreibung nicht um eine Schikanebetreibung handelt (in Abwandlung des alten Sprichworts «Zweier Zeugen Mund tut Wahrheit kund»).

57 Des Weiteren ist nicht zu übersehen, dass mit der vorgeschlagenen Regelung die ersten zwei Betreibungen konsequent so behandelt werden, «als ob» es sich um ungerechtfertigte Betreibungen handle. Betrachtet man die Gründe, die dazu führen können, dass eine Betreibung nicht fortgesetzt wird (vorne, Ziff. III), so scheint diese Vermutung nicht notwendigerweise gerechtfertigt. Dies gilt umso mehr, als es sich genau besehen nicht bloss um die «ersten zwei Betreibungen» handelt, sondern um sämtliche Betreibungen, die von den ersten zwei Gläubigern eingeleitet worden sind. Ein Beispiel mag dies verdeutlichen: Hat der Vermieter des Schuldners je eine Betreibung für den Mietzins der Monate Januar, Februar und März eingeleitet, und hat der Krankenversicherer des Schuldners ebenso drei Betreibungen für die Krankenkassenprämien der Monate Januar, Februar und März eingeleitet, so liegen gegen den Schuldner bereits sechs Betreibungen von zwei Gläubigern vor. Sofern noch keine dieser Betreibungen fortgesetzt wurde, werden diese Betreibungen einem Dritten nicht bekannt gegeben. Mit grosser Wahrscheinlichkeit vermittelt in einer solchen Konstellation der (leere) Betreibungsregisterauszug einen Eindruck, der 
nicht der Realität entspricht. ${ }^{110}$

Das erwähnte Beispiel mag ein wenig überzeichnet erscheinen; realitätsnäher ist wohl der Schuldner, der (wenn auch nur aus Nachlässigkeit) hin und wieder seine Forderungen auch nach Mahnung nicht begleicht, so dass er alle paar Monate betrieben wird. Sofern innerhalb einer Periode von sechs Monaten höchstens zwei Gläubiger die Betreibung eingeleitet haben und der Schuldner jeweils unter Betreibungsdruck doch noch (direkt an den Gläubiger) bezahlt, bevor es zur Fortsetzung kommt, kann sich auch dieser Schuldner einen reinen Betreibungsauszug verschaffen. Auch hier stimmt der vom Betreibungsregister vermittelte Informationsgehalt nicht mit der Realität überein: Die Warnung vor einem nur schleppend zahlenden Schuldner bliebe unter Umständen völlig aus. ${ }^{111}$

Schliesslich besteht die Gefahr, dass bei Personen mit häufig wechselndem Wohnsitz das Einsichtsrecht weitgehend ausgehebelt wird, da nur das Vorliegen von zwei Betreibungen innerhalb von sechs Monaten vor dem gleichen Betreibungsamt dazu führt, dass der Antrag auf Verweigerung der Bekanntgabe an Dritte abgelehnt wird. ${ }^{112}$ Aus pragmati-

$110 \overline{\text { Vgl. zu dieser Problematik auch Schmid (Fn. 29), }}$ Rz. 25.

111 Jean-Daniel Schmid, Die Löschung der Betreibung im Betreibungsregister nach erfolgtem Rückzug (Art. 8a Abs. 3 lit. c SchKG), AJP 2015, 610 ff., sieht diese Gefahr auch de lege lata, wenn der säumige Schuldner den Gläubiger überzeugen kann, die Betreibung zurückzuziehen.

112 Allerdings kann der Schuldner auch nach dem heute geltenden System durch einen Wohnsitzwechsel zu einem reinen Betreibungsregisterauszug kommen, da auf dem Auszug bloss die vom ausstellenden Amt geführten Betreibungen verzeichnet sind, schen Gründen lässt sich eine schematische Lösung nicht anders umsetzen - die Betreibungsämter haben keine Kenntnis von Betreibungen, die vor einem anderen Betreibungsamt eingeleitet wurden praktisch ist diese Folge sicherlich unerwünscht.

60 Unseres Erachtens ist dem Bericht zwar insofern beizupflichten, dass ungerechtfertigte Betreibungen gewöhnlicherweise als Einzelfälle auftreten und dass deshalb da, wo mehrere Gläubiger betreiben, eine gewisse Wahrscheinlichkeit für eine mangelnde Zahlungsfähigkeit oder Zahlungswilligkeit des Schuldners besteht, was die Aufführung der entsprechenden Betreibungen im Betreibungsregister rechtfertigt. Problematisch erscheint uns jedoch, dass der Gesetzesvorschlag den Umkehrschluss zu machen scheint, dass das Vorhandensein von höchstens zwei Betreibungen (oder von mehreren Betreibungen von maximal zwei Gläubigern) darauf hindeutet, dass diese Betreibungen ungerechtfertigt sind und deshalb auf Antrag des Schuldners nicht bekannt gegeben werden dürfen. Diese Annahme trifft nach unserer Meinung nicht zu. Der Gesetzesvorschlag schiesst daher einerseits übers Ziel hinaus, weil in zahlreichen Fällen auch die Bekanntgabe von gerechtfertigten Betreibungen unterdrückt werden könnte, und gleichzeitig ist er zu wenig weit gehend, weil er da keine Abhilfe schafft, wo eine ungerechtfertigte Betreibung von den Ausschlusskriterien nicht erfasst wird.

61 Die Regelung des Einsichtsrechts gemäss Art. 8a SchKG ist letztlich eine vom Gesetzgeber vorgenommene Abwägung der gegenläufigen Interessen des Schuldners einerseits und von (potentiellen) Gläubi- 
gern andererseits. Im Ergebnis bewirkt der neue Art. 8b E-SchKG eine Verschiebung der derzeit geltenden Balance zu Gunsten des Schuldners. Eine solche grundsätzliche Verschiebung dieser Balance war jedoch nicht das Ziel der parlamentarischen Initiative, und hier wurde auch nicht Handlungsbedarf geltend gemacht. ${ }^{113}$ Verbessert werden sollten vielmehr die Möglichkeiten des Schuldners, sich gegen ungerechtfertigte Betreibungen zur Wehr zu setzen, da die derzeit zur Verfügung stehenden Möglichkeiten oftmals nicht zielführend oder mit hohen Kosten und hohem Aufwand verbunden sind. ${ }^{114}$ Unseres Erachtens müsste ein Reformvorschlag denn auch viel eher hier, bei den festgestellten Defiziten, ansetzen, als dass versucht wird, anhand abstrakter Kriterien ungerechtfertigte Betreibungen vom Einsichtsrecht auszuschliessen.

62 Es stellt sich daher die Frage, ob dem Ansinnen der parlamentarischen Initiative nicht anderweitig nachgekommen und dem Schuldner ein Mittel zur Verfügung gestellt werden kann, mit dem er einen unliebsamen Betreibungsregistereintrag - sofern dieser denn zu Unrecht besteht - relativ einfach, rasch und kostengünstig löschen lassen kann.

113 Es hätte durchaus auch eine radikale Änderung des Einsichtsrechts gefordert werden können, etwa in dem Sinne, dass vom Einsichtsrecht nur solche Betreibungen erfasst werden, in denen der Zahlungsbefehl rechtskräftig geworden ist oder in denen der Gläubiger zumindest Anstrengungen unternimmt, den Rechtsvorschlag zu beseitigen (vgl. hierzu Schmid [Fn. 29], Rz. 24). Dies quasi als Korrelat zur Möglichkeit des Gläubigers, voraussetzungslos einen Zahlungsbefehl (und damit einen Eintrag im Betreibungsregister) zu erwirken.

114 So auch explizit der Bericht der Kommission (Fn. 3), 3214.

\section{Vorschlag der Autoren: Ausbau der Klage nach Art. 85 SchKG}

\section{Formulierungsvorschlag}

63 Prüfenswert erscheint den Autoren ein Ausbau der Klage nach Art. 85 SchKG. Gemäss heute geltender Fassung kann der Schuldner mit dieser Klage die Aufhebung (oder Einstellung) der Betreibung erwirken, wenn er mittels Urkunden beweisen kann, dass die betriebene Forderung getilgt oder gestundet ist oder - so die Rechtsprechung ${ }^{115}$ - nie bestand. Vermag der Betriebene darzutun, dass die Forderung bereits bei Einleitung der Betreibung getilgt war, so erreicht er damit, dass die Betreibung Dritten nicht mehr zur Kenntnis gebracht wird. ${ }^{116}$ Wenn jedoch der Schuldner für eine Forderung betrieben worden ist, die gar nie bestanden hat, wird er dies kaum je durch Urkunden beweisen können.117 Hier liegt unseres Erachtens Potenzial für eine Verbesserung, die den Informationswert des Betreibungsregisters erhöht und den berechtigten Interessen des zu Unrecht Betriebenen Rechnung trägt.

64 Anstatt den Schuldner ausschliesslich auf den strikten Urkundenbeweis zu verweisen, wäre der Anwendungsbereich der Klage nach Art. 85 SchKG so zu erweitern, dass eine durch Rechtsvorschlag zum Stillstand gekommene Betreibung auf Begehren des Schuldners aufgehoben wird, wenn der Gläubiger den Bestand der Forderung nicht glaubhaft macht. Konkret schlagen wir folgenden Wortlaut für den (revidierten) Art. 85 SchKG vor:

115 BGE 140 III 41 E. 3.3.1.

${ }^{116}$ Siehe vorne, Ziff. 4.

117 Ausser etwa durch Vorlage eines negativen gerichtlichen Feststellungsentscheids oder eines negativen Schuldbekenntnisses (BGE 140 III 41, E. 3.3.2). 


\section{Art. 85 SchKG (Entwurf)}

1 Beweist der Betriebene durch Urkunden, dass die Schuld samt Zinsen und Kosten getilgt ist, nie bestanden hat oder gestundet ist, so kann er jederzeit vom Gericht des Betreibungsortes in den ersteren beiden Fällen die Aufhebung, im letzteren Fall die Einstellung der Betreibung verlangen.

${ }^{2}$ Ist die Betreibung durch Rechtsvorschlag eingestellt, und ist dessen Beseitigung nicht Gegenstand eines anderen hängigen Verfahrens, so kann der Betriebene vom Gericht des Betreibungsortes die Aufhebung der Betreibung verlangen, wenn

a. er den Gläubiger erfolglos aufgefordert hat, die Betreibung innert 30 Tagen zurückzuziehen, und

b. der Gläubiger nicht glaubhaft macht, dass seine Forderung besteht und fällig ist.

\section{Abs. 1 des Entwurfs}

65 Absatz 1 des Entwurfs entspricht dem bisher geltenden Art. 85 SchKG. Die einzige Abweichung zum bisherigen Wortlaut besteht in der Klarstellung, dass nicht bloss der urkundliche Nachweis der Tilgung zur Aufhebung der Betreibung führt, sondern auch der urkundliche Nachweis des Nichtbestands der Forderung. Damit wird nur die höchstrichterliche Rechtsprechung zum geltenden Art. 85 SchKG kodifiziert.

Im Sinne der neueren Rechtsprechung ${ }^{118}$ kann die Aufhebung oder Einstellung nicht nur bei laufender Betreibung verlangt werden, sondern auch dann, wenn die Betreibung zufolge erhobenen (und nicht beseitigten) Rechtsvorschlags zum Stillstand gekommen ist. Auf eine diesbezügliche Verdeutlichung wird im Entwurf verzichtet.

$118 \overline{\text { BGE } 140 \text { III } 41 \text { E. 3.2.3. }}$
67 Die Aufhebung der Betreibung bewirkt nicht ohne weiteres, dass die Betreibung Dritten nicht mehr zur Kenntnis gebracht wird. Im Sinne der bisherigen Rechtsprechung ${ }^{119}$ wird das Einsichtsrecht Dritter in Bezug auf die fragliche Betreibung nur dann ausgeschlossen, wenn die Forderung bereits im Zeitpunkt der Einleitung der Betreibung getilgt oder gestundet war (oder gar nie bestanden hat). ${ }^{120}$ Hieran ist festzuhalten.

\section{Abs. 2 des Entwurfs}

68 Die neue Klage nach Art. 85 Abs. 2 Entwurf ist nur zulässig, wenn die Betreibung durch Rechtsvorschlag zum Stillstand gekommen ist. Ist dies nicht der Fall, hat also der Schuldner gar nicht erst Rechtsvorschlag erhoben, oder wurde der Rechtsvorschlag beseitigt, so impliziert dies, dass der Schuldner die Forderung nicht bestritten hat oder ihr nichts entgegensetzen konnte. Unter diesen Umständen erscheint es gerechtfertigt, dass der Schuldner die Aufhebung (bzw. Einstellung) der Betreibung nur unter den strengeren Kriterien gemäss Art. 85 Abs. 1 Entwurf (Urkundenbeweis bezüglich Tilgung, Nichtbestand oder Stundung der Forderung) verlangen kann.

69 Unzulässig ist die Klage nach Art. 85 Abs. 2 Entwurf, wenn die Beseitigung des Rechtsvorschlags bereits Gegenstand eines anderen Verfahrens bildet, beispielsweise eines vom Gläubiger eingeleiteten Anerkennungsprozesses oder Rechtsöffnungsverfahrens. In diesem Fall unternimmt der Gläubiger bereits alles, um die Betreibung voranzutreiben, und der Betriebene soll dies nicht verhindern können.

119 BGE 125 III 334 E. 3.

120 Siehe vorne, Ziff. 4. 
7o Für ein erfolgreiches Vorgehen nach Art. 85 Abs. 2 Entwurf ist erforderlich, dass der Schuldner den Gläubiger vergeblich aufgefordert hat, die Betreibung binnen 30 Tagen zurückzuziehen. Zieht der Gläubiger die Betreibung binnen dieser Frist nicht zurück, so wird er durch die Klage gemäss Abs. 2 unseres Entwurfs gezwungen, seine Forderung glaubhaft zu machen. Dies erscheint uns jedoch nicht unbillig. Wenn der Gläubiger nicht einmal in der Lage ist, wenigstens Anhaltspunkte für den Bestand seiner Forderung darzutun, dann soll der Betriebene auch nicht durch eine Betreibung belastet werden. Da die Klage (auch in der vorgeschlagenen Ausgestaltung) bloss betreibungsrechtliche Wirkung hat, stünde es dem Gläubiger frei, im Falle seines Unterliegens die Forderung erneut in Betreibung zu setzen; er erlitte keinen materiellen Rechtsverlust und würde somit auch nicht gezwungen werden, vorzeitig, zu einem vom Schuldner bestimmten Zeitpunkt, seinen Anspruch durchzusetzen. Zudem stünde es dem Gläubiger frei, auch nach Einleitung des Verfahrens durch den Schuldner die Betreibung ohne Rechtsverlust zurückzuziehen; diesfalls entfiele das Rechtsschutzinteresse des Schuldners an der Klage nach Art. 85 Abs. 2 Entwurf.

Die Klage nach Art. 85 Abs. 2 Entwurf weist Parallelen zum Verfahren der provisorischen Rechtsöffnung auf: Beiden Verfahren ist gemein, dass für ein Obsiegen des Gläubigers nicht erforderlich ist, dass er seinen materiellen Anspruch beweist. Während jedoch im Rechtsöffnungsverfahren der Gläubiger (als Kläger) eine Schuldanerkennung vorzulegen hat, kann er im neuen Verfahren nach Art. 85 Abs. 2 Entwurf seinen Anspruch (als Beklagter) auch anderweitig glaubhaft machen. Der Schuldner kann die glaubhaft gemachte Forderung seinerseits durch glaubhaft gemachte Einreden und Einwendungen entkräften.

72 Für den beklagten Gläubiger stellt sich die Frage, was ihm ein Obsiegen in einem Verfahren nach Art. 85 Abs. 2 Entwurf effektiv bringt: Der Aufwand, sich vor Gericht gegen den klagenden Schuldner $\mathrm{zu}$ verteidigen, nur um die eingeleitete Betreibung «am Leben zu erhalten», dürfte dem Gläubiger in der Regel unverhältnismässig erscheinen. Hinzuweisen ist jedoch darauf, dass derjenige beklagte Gläubiger, der im Besitz eines Rechtsöffnungstitels gegen den Schuldner ist, Widerklage erheben und die Erteilung der Rechtsöffnung verlangen kann, denn die Voraussetzungen für das Erheben einer Widerklage sind gegeben: Sowohl für die Klage nach Art. 85 Abs. 2 Entwurf als auch für das Rechtsöffnungsverfahren ist das Gericht am Betreibungsort örtlich zuständig, ${ }^{121}$ und beide Klagen sind im summarischen Verfahren zu beurteilen. ${ }^{122}$ Obwohl die ZPO die Widerklage nur für das ordentliche Verfahren ausdrücklich vorsieht, ${ }^{123}$ ist anerkannt, dass eine Widerklage grundsätzlich auch im summarischen Verfahren zulässig ist, sofern sie das Verfahren nicht wesentlich verzögert. ${ }^{124}$ Falls der

121 Art. 84 Abs. 1 SchKG und Art. 85 Abs. 2 Entwurf.

122 Art. 251 lit. a und lit. c ZPO sowie der unveränderte Randtitel von Art. 85 Entwurf.

123 Art. 224 ZPO.

124 Botschaft zur Schweizerischen Zivilprozessordnung vom 28. Juni 2006, BBl 2006 7221, 7350; Chevalier, in Sutter-Somm/Hasenböhler/Leuenberger (Hrsg.), Kommentar zur Schweizerischen Zivilprozessordnung (ZPO), 2. Aufl., Zürich/Basel/Genf 2013, Art. 252 N 23 ff.; Willisegger, in Spühler/Tenchio/Infanger (Hrsg.), Basler Kommentar, Schweizerische Zivilprozessordnung, Basel 2010 (nachfolgend «BSK-ZPO-Bearbeiter»), 
widerklagende Gläubiger seinen Rechtsöffnungstitel ohne Verzug an der mündlichen Verhandlung vorlegt oder, falls das Verfahren schriftlich geführt wird, ihn mit seiner schriftlichen Stellungnahme innert Frist einreicht, ist kaum mit einer wesentlichen Verfahrensverzögerung zu rechnen. Obsiegt der Gläubiger mit seiner Widerklage, wird ihm Rechtsöffnung erteilt.

Die Möglichkeit eines solchen Verfahrensausgangs stellt für den Schuldner natürlich ein Risiko dar: Obsiegt der Gläubiger mit seinem widerklageweisen Rechtsöffnungsgesuch, wird das durch den Rechtsvorschlag zum Stillstand gekommene Betreibungsverfahren wieder in Gang gesetzt. Salopp gesagt erweist sich die Klage nach Art. 85 Abs. 2 Entwurf in einem solchen Fall für den Schuldner als «Eigentor». Diesem Risiko kommt die gewünschte Funktion eines Korrektivs zu: Der Schuldner wird die Klage auf Aufhebung der Betreibung nicht leichtfertig anheben, wenn er davon ausgehen muss, dass der Gläubiger für seine Forderung «gute Karten» (sprich, einen Rechtsöffnungstitel) in der Hand hat. Selbstverständlich steht es dem Gläubiger in einer solchen Konstellation auch frei, dem Schuldner die Grundlage für ein Verfahren nach Art. 85 Abs. 2 Entwurf zu entziehen, indem er vor Ablauf der 30-Tagesfrist von Art. 85 Abs. 2 lit. a Entwurf ein Rechtsöffnungsverfahren einleitet.

74 Ist der Gläubiger zwar der festen Überzeugung, dass seine Forderung besteht, verfügt er jedoch nicht über einen Rechtsöffnungstitel, so würde er mit einem widerklageweisen erhobenen

Art. 224 N 73; BSK-ZPO-Mazan, Art. 253 N 20.
Rechtsöffnungsgesuch nicht durchdringen. Auch wenn er seine Forderung anderweitig glaubhaft machen könnte (bspw. durch E-Mail-Korrespondenz) und er im Verfahren nach Art. 85 Abs. 2 Entwurf obsiegen würde, würde das Betreibungsverfahren damit nicht vorangetrieben. Der Gläubiger hätte bloss erreicht, dass die Betreibung nicht aufgehoben wird. In dieser Konstellation ist der Gläubiger daher gut beraten, vor Ablauf der 30-Tagesfrist nach Art. 85 Abs. 2 lit. a Entwurf entweder die Betreibung zurückzuziehen oder aber ein Verfahren zur Durchsetzung seiner Forderung einzuleiten. Unserer Ansicht nach würde der Gläubiger damit nicht ungebührlich unter Druck gesetzt:

- Ist es dem Gläubiger aus irgendwelchen Gründen noch nicht möglich, Klage einzuleiten, so kann er die Betreibung ohne Rechtsverlust wieder zurückziehen. Hat der Gläubiger die Betreibung primär zur Unterbrechung der Verjährung eingeleitet, so bleibt die verjährungsunterbrechende Wirkung auch bei einem Rückzug der Betreibung erhalten. ${ }^{125}$

- Erscheint dem Gläubiger die 30Tagesfrist zur Einreichung einer Klage zu knapp, so kann er die Frist mit der Einreichung eines nicht oder nur knapp begründeten Schlichtungsgesuchs wahren und danach, in der Zeit bis zur Schlichtungsverhandlung und während der dreimonatigen Gültigkeitsdauer der Klagebewilligung, ${ }^{126}$ die detaillierte Klageschrift verfassen.

\footnotetext{
125 BSK-OR-Däppen (Fn. 59), Art. 135 N 6; BSKSchKG-Kofmel Ehrenzeller (Fn. 39), Art. 68 $\mathrm{N} 48$.

126 Art. 209 Abs. 3 ZPO.
} 
- Will der Gläubiger jedoch gegen den Willen des Schuldners eine eingeleitete Betreibung stehen lassen, aber gleichwohl nichts zur Durchsetzung seiner Forderung unternehmen, so erscheint es unseres Erachtens gerechtfertigt, dass der Gläubiger sich dieses Recht im Verfahren nach Art. 85 Abs. 2 Entwurf erstreiten muss, indem er seine Forderung glaubhaft macht, auch wenn ihn dies im Betreibungsverfahren nicht weiter voranbringt.

Handelt es sich bei der fraglichen Betreibung um eine offensichtlich ungerechtfertigte Betreibung, und weigert sich der Gläubiger auf Aufforderung des Schuldners hin, die Betreibung zurückzuziehen, stellt die Aufhebungsklage gemäss Art. 85 Abs. 2 Entwurf ein taugliches Mittel dar, den Betreibungsregisterauszug zu bereinigen. Kann der Betreibende nicht glaubhaft machen, dass seine Forderung besteht und fällig ist, so wird die Betreibung aufgehoben. Dies führt allerdings nicht zwingend in jedem Fall zum Ausschluss des Einsichtsrechts: Im Sinne der bisherigen Rechtsprechung zu den bisherigen Rechtsbehelfen des Schuldners ${ }^{127}$ würde die aufgehobene Betreibung Dritten (nur) dann nicht mehr zur Kenntnis gebracht, wenn sich aus dem Aufhebungsurteil ergibt, dass die Forderung bei Einleitung der Betreibung nicht bestand und nicht fällig war. Dies dürfte indes bei einem gutheissenden Aufhebungsurteilen regelmässig der Fall sein: Vermag der Gläubiger nicht glaubhaft zu machen, dass die betriebene Forderung gegenwärtig existiert, ist damit in der Regel auch glaubhaft, dass die Forderung bereits bei Einleitung der Betreibung

127 Vgl. BGE 125 III 334 E. 3. nicht bestand. Einzig in den Fällen, in denen der Gläubiger zwar glaubhaft macht, dass die Forderung bei Einleitung der Betreibung bestand, der Schuldner jedoch das zwischenzeitliche Erlöschen der Forderung glaubhaft machen kann, erginge ein gutheissendes Aufhebungsurteil, während die betreffende Betreibung weiterhin Dritten zur Kenntnis gebracht werden dürfte. ${ }^{128}$ Unter dem Blickwinkel des Informationsgehalts des Betreibungsregisters wäre dies gerechtfertigt, denn die Betreibung wurde offenbar zu Recht eingeleitet, weshalb sie auch nach ihrer Aufhebung weiterhin für Dritte einsehbar sein soll.

76 Da die Klage im summarischen Verfahren behandelt wird und der Gebührentarif SchKG zur Anwendung kommt, würde auch dem Bedürfnis nach Raschheit und niedrigen Kosten Rechnung getragen. Durch eine konsequente Kautionierung der (massvollen) Verfahrenskosten können die Gerichte zudem verhindern, dass der Schuldner - insbesondere der Schuldner mit zweifelhafter Zahlungsfähigkeit - allzu leicht von dieser Klage Gebrauch machen kann.

\section{Zusammenfassung}

77 Dem Betreibungsregister kommt in der Praxis eine wichtige Rolle bei der Überprüfung der Kreditwürdigkeit zu. Damit es diese Rolle erfüllen kann, sollten alle Vorgänge, aber eben auch nur solche Vorgänge, Dritten zur Kenntnis gebracht werden, die einen Rückschluss auf die

${ }_{128}$ Diese Konstellation entspräche der Situation gemäss heutigem Recht, wenn der Schuldner nach Art. 85 SchKG vorgeht und den urkundlichen Nachweis erbringt, dass die Forderung nach Einleitung der Betreibung getilgt wurde (siehe vorne Ziff. 4). 
Zahlungsfähigkeit oder -willigkeit des Betriebenen erlauben. Die Existenz von Einträgen im Betreibungsregister kann für den Betroffenen auch dann erhebliche Nachteile mit sich bringen, wenn es sich bloss um einzelne Betreibungen über geringfügige Beträge handelt. Einträge, die keinen Informationsgehalt bezüglich der Kreditwürdigkeit des Betriebenen aufweisen, sollten Dritten nicht bekannt gegeben werden. Nach geltendem Recht verfügt der Betriebene zwar über eine ganze Palette an Rechtsbehelfen, um sich gegen eine Betreibung zur Wehr zu setzen. Wenn es aber darum geht, einen ungerechtfertigten Betreibungsregistereintrag löschen zu lassen, sind diese Rechtsbehelfe häufig mit hohen Kosten verbunden und es dauert sehr lange, bis ein ungerechtfertigter Eintrag tatsächlich gelöscht wird.

Die Reformbemühungen, diese für den Betriebenen oftmals unbefriedigende Situation zu verbessern, sind zu begrüssen. Der auf die parlamentarische Initiative Abate hin ausgearbeitete Gesetzesvorschlag zur Revision des SchKG vom 19. Februar 2015 soll hier Abhilfe schaffen. Die Autoren stimmen dem Vorschlag insofern zu, als der Anwendungsbereich der Klage nach Art. 85a SchKG dahingehend ausgeweitet werden soll, dass die Klage auch dann erhoben werden kann, wenn die Betreibung zufolge erhobenen Rechtsvorschlags eingestellt ist. Weniger überzeugend erscheint jedoch die vorgesehene Beschränkung der Bekanntgabe von Betreibungen an Dritte. Nach Auffassung der Autoren trägt die geplante Regelung nicht zur Verbesserung des Informationswerts des Betreibungsregisters bei. Der vom Gesetzesvorschlag verfolgte Ansatz, ungerechtfertigte Betrei- bungen mittels schematischer Kriterien zu erfassen und vom Einsichtsrecht auszunehmen, ist unseres Erachtens nicht zielführend.

79 Im Sinne eines Beitrags zur Diskussion regen die Autoren an, einen Ausbau der Klage nach Art. 85 SchKG zu prüfen. Es wird vorgeschlagen, den bisherigen Anwendungsbereich der Klage dahingehend zu erweitern, dass mit ihr eine Betreibung aufgehoben werden kann, wenn der Betriebene den Betreibenden vorgängig erfolglos zum Rückzug der Betreibung aufgefordert hat und der Gläubiger nicht glaubhaft macht, dass die Betreibungsforderung besteht und fällig ist. Ist der Schuldner mit seiner Klage erfolgreich, führt dies in der Regel, von Ausnahmekonstellationen abgesehen, dazu, dass die betreffende Betreibung vom Einsichtsrecht Dritter ausgeschlossen wird. Wer eine Betreibung einleitet, sollte zumindest in der Lage sein, den Bestand der betriebenen Forderung glaubhaft zu machen. Ist er dazu nicht fähig, so rechtfertigt sich eine Bekanntgabe der Betreibung gegenüber Dritten aus Sicht des Informationsgehalts des Betreibungsregisters nicht. Da die Klage nach Art. 85 SchKG auch nach unserem Vorschlag ausschliesslich betreibungsrechtliche Wirkungen hat, bleibt dem Gläubiger der Zivilweg offen. Die Befürchtung, dass er durch die Klage unter der Gefahr, seinen Anspruch endgültig zu verlieren, zur vorzeitigen Beweisführung gezwungen wird, manifestiert sich daher nicht. Das berechtigte Interesse des Betriebenen, dass die Betreibung einer nicht glaubhaft gemachten Forderung Dritten nicht bekannt gegeben wird, überwiegt. 